\title{
A Population Classification Evolution Algorithm for the Parameter Extraction of Solar Cell Models
}

\author{
Yiqun Zhang, ${ }^{1,2}$ Peijie Lin, ${ }^{1,2}$ Zhicong Chen, ${ }^{1,2}$ and Shuying Cheng ${ }^{1,2}$ \\ ${ }^{1}$ College of Physics and Information Engineering and Institute of Micro-Nano Devices and Solar Cells, Fuzhou University, \\ Fuzhou 350116, China \\ ${ }^{2}$ Jiangsu Collaborative Innovation Center of Photovoltaic Science and Engineering, Changzhou 213164, China \\ Correspondence should be addressed to Shuying Cheng; sycheng@fzu.edu.cn
}

Received 11 May 2016; Revised 21 June 2016; Accepted 22 June 2016

Academic Editor: Tamer Khatib

Copyright (C) 2016 Yiqun Zhang et al. This is an open access article distributed under the Creative Commons Attribution License, which permits unrestricted use, distribution, and reproduction in any medium, provided the original work is properly cited.

To quickly and precisely extract the parameters for solar cell models, inspired by simplified bird mating optimizer (SBMO), a new optimization technology referred to as population classification evolution (PCE) is proposed. PCE divides the population into two groups, elite and ordinary, to reach a better compromise between exploitation and exploration. For the evolution of elite individuals, we adopt the idea of parthenogenesis in nature to afford a fast exploitation. For the evolution of ordinary individuals, we adopt an effective differential evolution strategy and a random movement of small probability is added to strengthen the ability to jump out of a local optimum, which affords a fast exploration. The proposed PCE is first estimated on 13 classic benchmark functions. The experimental results demonstrate that PCE yields the best results on 11 functions by comparing it with six evolutional algorithms. Then, PCE is applied to extract the parameters for solar cell models, that is, the single diode and the double diode. The experimental analyses demonstrate that the proposed PCE is superior when comparing it with other optimization algorithms for parameter identification. Moreover, PCE is tested using three different sources of data with good accuracy.

\section{Introduction}

The rising cost of fossil fuels, atmospheric pollution, and global energy shortage have prompted the development and use of renewable energy $[1,2]$. PV (photovoltaic) systems such as solar cells have recently received significant attention with characteristics of renewability, clean-type, convenience, and low noise technique $[1,3,4]$. PV systems usually operate in outdoor environment and their PV arrays are prone to deteriorate and may even undergo various faults due to harsh weather condition and aging, which greatly affect the solar energy utilization efficiency and even cause safety issues. Therefore, in order to optimize PV systems, it is crucial to evaluate the actual behavior of PV arrays in operation through accurate modeling based on experimental data. Numerous mathematical models have been proposed to clarify the characteristic of a PV system under different operating conditions. However, in practical terms, two solar cell models are the most frequently used: the single and double diode models $[3,5]$. Although the double diode model can achieve more precise results than the single diode model, the ability to achieve an adequate concession between simplified and precise results in the single diode model is a more preferable option [6]. Accurate parameters of a mathematical model is crucial to simulate, estimate, and optimize solar cell systems. Therefore, it is necessary to consider parameters identification with a feasible optimization approach [7].

The techniques utilized to identify the parameters of PV models in the literature can be divided in two groups: deterministic techniques and heuristic techniques [2]. Deterministic techniques, that is, least squares [8], Lambert $W$ functions [9], and iterative curve fitting [10], force some model constraints like convexity and differentiability which must be exactly used. Thus, these deterministic methods drastically depend on initialization and can also be easy to fall into a local optimum [2]. Recently, based on the theory of reproduction and evolution of different biological populations, many heuristic technologies have been proposed to deal with the parameter estimation issues of solar cells such as particle swarm optimization (PSO) [11, 12], genetic algorithms (GA) [13-15], differential evolution (DE) [4, 16-18], pattern search (PS) [19], simulated annealing 
(SA) [20], harmony search (HS) [21], and artificial bee swarm optimization (ABSO) [3]. Although heuristic methods present a higher probability of obtaining a global solution in comparison with deterministic ones, they have important limits [13]. In case of GA and PSO, they maintain a trend that concentrates toward local optima, since their elitist mechanism forces premature convergence [2]. Such behavior becomes worse when the optimization algorithm faces multimodal functions. On the other hand, due to the fact that SA and HS are single-searcher algorithms, their performance is sensitive to the starting point of the search, having a lower probability to localize the global minimum in multimodal problems than population algorithms such as GA and PSO [2]. Therefore, GA, PSO, SA, and HS present bad performance when they are applied to multimodal and noisy objective functions. Therefore, there is the possibility to yield better performance with more capable algorithms.

In biological populations, birds have around 10,000 living species, which are the most species of tetrapod vertebrates [22]. In birds, the courtship behavior is innate. During the mating season, a bird tries to find spouse(s) with good genes for raising a brood that can survive for a longer time, which is similar to that of a search for the optimal solution. To develop a more capable heuristic optimization algorithm, Askarzadeh and Rezazadeh [22] propose a bird mating optimizer (BMO). In BMO algorithm, there are four breeding strategies: monogamy, polygamy, polyandry, and promiscuity. Experimental results indicate the superior performance of BMO. However, there are two main drawbacks in BMO: (1) numerous types of birds and (2) numerous numbers of tunable parameters. To overcome these disadvantages, Askarzadeh and dos Santos Coelho [6] propose a simplified $\mathrm{BMO}$ algorithm (SBMO). In SBMO algorithm, all birds in the population are ranked according to their objective function values such that the bird with the best objective function value is ranked first. Then, these birds are divided into three types for breeding based on their rank. These three breeding strategies are parthenogenesis, monogamy, and polygamy, respectively. The SBMO algorithm reduces the computational complexity and yields good performance on PV modules.

However, SBMO has a disadvantage of slow convergence. Firstly, the disturbance coefficient in Type 1 (parthenogenesis) is very small, which means the exploitation space is very limited. Thus, it is not easy to find a better solution. Secondly, in Type 2 (monogamy), a female mating bird is randomly selected from Type 1 . Therefore, the selected female mating bird may be not the optimal. The convergence speed of Type 2 is limited. Thirdly, Type 3 (polygamy) plays a similar role to Type 2, which means SBMO has no effective strategy to avoid trapping in a local optimum.

The parameter estimation method should be reliable, accurate, and fast, for a range of different types of cells estimation. To further improve the efficiency and reliability of parameters identification of photovoltaic models, here, we propose a new optimization technique referred to as population classification evolution (PCE) to overcome the slow convergence of SBMO. In PCE, we divide the population into two categories, that is, elite and ordinary. The elite are the parthenogenesis corresponding to the neighborhood-based search known as the local search strategy to afford exploitation. In the elite evolution strategy, we magnify the exploitation space to accelerate convergence. In the ordinary, we employ DE mutation "DE/best/1" operator to guide an individual to be close to the global optimum. Then, a crossover operator following mutation is employed to increase the diversity of the population. In addition, we add a random movement of small probability for the evolution of ordinary individuals to further increase the diversity of the population and overcome the premature convergence. Obviously, the ordinary evolution is corresponding to the global search to afford exploration. To estimate the performance of PCE, it is first compared with five well-known evolutional algorithms (EAs), SBMO, cuckoo search (CS), artificial bee colony $(A B C)$, improved adaptive differential evolution (IADE), and self-adapting control parameters in differential evolution (called jDE) on 13 classic benchmark functions. And a startof-the-art evolutional algorithm called generalized oppositional teaching learning based optimization (GOTLBO) is also adopted to compare with PCE. PCE has a faster convergence speed and higher accuracy than these six EAs on most benchmark functions through emulation. In addition, PCE is applied to extract the parameters of both the single and double diode model. The emulation data indicate that the proposed PCE is superior when comparing it with other parameter extraction methods. Moreover, PCE is tested using three different sources of data with good accuracy.

Organization of the remainder of the paper is as follows. For Section 2, solar cell identification is depicted. In Section 3, the SBMO algorithm is depicted. Section 4 specifies the implementation of our proposed PCE. In Section 5, PCE is estimated on 13 classic benchmark functions. Then, in Section 6, PCE is applied to extract the parameters of PV models. The conclusions are depicted in Section 7.

\section{Problem Statement}

2.1. Solar Cell Models. An accurate mathematical model describing the electrical characteristics of solar cells is needed in advance. Some equivalent circuit models are used to simulate the current-voltage $(I-V)$ behavior for solar cells, but only two models are employed in practice: the double diode model and the single diode model. In this subsection, these models are tersely discussed.

2.1.1. Double Diode Model. Under the illumination, the ideal solar cell model is a photogenerated current source that is shunted by a rectifier diode [3, 13, 21]. Figure 1 shows the equivalent circuit of the double diode model. According to Kirchhoff's law of electric current and the Shockley equation, the $I-V$ relationship is formulated as

$$
\begin{aligned}
I_{t}= & I_{\mathrm{ph}}-I_{\mathrm{d} 1}-I_{\mathrm{d} 2}-I_{\mathrm{sh}} \\
= & I_{\mathrm{ph}}-I_{\mathrm{sd} 1}\left[\exp \left(\frac{q\left(V_{t}+R_{s} I_{t}\right)}{n_{1} k T}\right)-1\right] \\
& -I_{\mathrm{sd} 2}\left[\exp \left(\frac{q\left(V_{t}+R_{s} I_{t}\right)}{n_{2} k T}\right)-1\right]-\frac{V_{t}+R_{s} I_{t}}{R_{\mathrm{sh}}},
\end{aligned}
$$


where $I_{t}$ is the terminal current; $I_{\mathrm{ph}}$ denotes the photogenerated current; $I_{\mathrm{d} 1}$ and $I_{\mathrm{d} 2}$ are the first and second diode currents; and $I_{\mathrm{sh}}$ is the shunt resistor current. $I_{\mathrm{sd} 1}$ and $I_{\mathrm{sd} 2}$ denote the diffusion and saturation currents, respectively; $V_{t}$ denotes the terminal voltage; $R_{s}$ and $R_{\mathrm{sh}}$ denote the series and shunt resistances, respectively; $q$ is the electronic charge; $T$ $(\mathrm{K})$ denotes the cell temperature; $k$ is the Boltzmann constant; $n_{1}$ and $n_{2}$ denote the diffusion and recombination diode ideality factors, respectively.

This double diode model includes seven unknown parameters $\left(I_{\mathrm{ph}}, I_{\mathrm{sd} 1}, I_{\mathrm{sd} 2}, R_{s}, R_{\mathrm{sh}}, n_{1}\right.$, and $\left.n_{2}\right)$ to be estimated from (1). To reflect the solar cell performance as well as that of the real system, it is crucial to acquire an accurate parameters' identification.

2.1.2. Single Diode Model. Figure 2 shows the single diode model that is widely employed for modeling solar cells due to its simplicity. It is calculated as follows:

$$
I_{t}=I_{\mathrm{ph}}-I_{\mathrm{sd}}\left[\exp \left(\frac{q\left(V_{t}+R_{s} I_{t}\right)}{n k T}\right)-1\right]-\frac{V_{t}+R_{s} I_{t}}{R_{\mathrm{sh}}},
$$

where $I_{\text {sd }}$ is reverse saturation current of diode and $n$ is the diode ideality factor.

For this model, five parameters $\left(I_{\mathrm{ph}}, I_{\mathrm{sd}}, R_{s}, R_{\mathrm{sh}}\right.$, and $\left.n\right)$ would be estimated in (2).

2.2. Objective Function. The unknown parameters are obtained from the $I-V$ data with an optimization algorithm. In this optimization method, each solution is denoted by a vector, $x$, where $x=\left[\begin{array}{lllllll}I_{\mathrm{ph}} & I_{\mathrm{sd} 1} & I_{\mathrm{sd} 2} & R_{s} & R_{\mathrm{sh}} & n_{1} & n_{2}\end{array}\right]$ for the double diode model and $x=\left[\begin{array}{lllll}I_{\mathrm{ph}} & I_{\mathrm{sd}} & R_{\mathrm{s}} & R_{\mathrm{sh}} & n\end{array}\right]$ for the single diode model. For expressing the objective function to be optimized, (1) and (2) are altered as follows:

$$
\begin{aligned}
f\left(V_{t}, I_{t}, x\right)= & I_{t}-I_{\mathrm{ph}} \\
& +I_{\mathrm{sd} 1}\left[\exp \left(\frac{q\left(V_{t}+R_{s} I_{t}\right)}{n_{1} k T}\right)-1\right] \\
& +I_{\mathrm{sd} 2}\left[\exp \left(\frac{q\left(V_{t}+R_{s} I_{t}\right)}{n_{2} k T}\right)-1\right] \\
& +\frac{V_{t}+R_{s} I_{t}}{R_{\mathrm{sh}}}, \\
f\left(V_{t}, I_{t}, x\right)= & I_{t}-I_{\mathrm{ph}}+I_{\mathrm{sd}}\left[\exp \left(\frac{q\left(V_{t}+R_{s} I_{t}\right)}{n k T}\right)-1\right] \\
& +\frac{V_{t}+R_{s} I_{t}}{R_{\mathrm{sh}}} .
\end{aligned}
$$

During the optimization process, we adopt the RMSE as the objective function $[1,3]$ to reflect the difference between the real data and simulated data, which is defined by

$$
F(x)=\sqrt{\frac{1}{N} \sum_{i=1}^{N}\left(f_{i}\left(V_{t}, I_{t}, x\right)\right)^{2}},
$$

where $N$ is the number of the simulated data.

For this optimization case, the objective function $F(x)$ in (4) would be minimized.

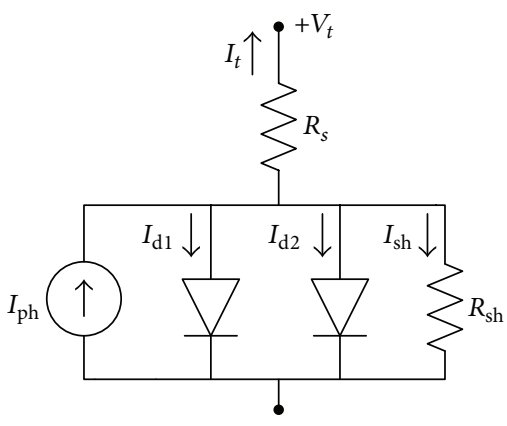

Figure 1: Single diode model.

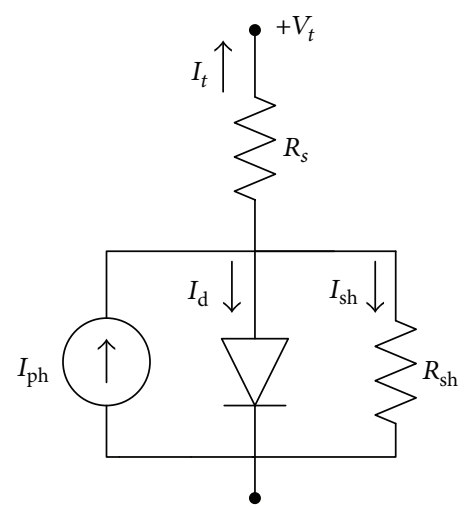

FIGURE 2: Double diode model.

\section{The Simplified Bird Mating Optimizer}

In SBMO [6], the birds are ranked according to their objective function values such that the bird with the best objective function value is ranked first. Then, these birds are divided into three types for breeding based on their rank.

Type 1. The birds of this type are called females that have better objective function values than others. The number of these birds $\left(N_{1}\right)$ is determined by

$$
N_{1}=\operatorname{round}\left(\frac{N P}{10}\right) \text {. }
$$

For breeding, female birds employ the following expression based on the idea of parthenogenesis [6,23-25]:

$$
\begin{aligned}
& \text { if rand }>\text { rand } \\
& \qquad v_{i}=x_{i}+\frac{\text { rand } \cdot(\text { rand }- \text { rand })}{10} \cdot x_{i} \\
& \text { else } \\
& \qquad v_{i}=x_{i}
\end{aligned}
$$

end,

where $x_{i}$ is the target vector related to the bird, $v_{i}$ is the mutant vector related to the bird's brood, and rand is a random number within $[0,1]$ and it is worthwhile mentioning that the random numbers are different. 
Type 2. The birds of this type are referred to as male birds. The number of these birds $\left(N_{2}\right)$ is determined by

$$
N_{2}=\operatorname{round}\left(\frac{7 \cdot N P}{10}\right)
$$

For breeding, male birds are interested in mating with one female bird:

$$
v_{i}=x_{i}+\operatorname{rand} \cdot\left(x_{s}-x_{i}\right)
$$

where $x_{s}$ is a target vector that is randomly selected from Type 1 for the interesting mate of the male bird.

Type 3. The birds of this type are referred to as male birds. The number of these birds $\left(N_{3}\right)$ is determined by

$$
N_{3}=N P-N_{1}-N_{2} \text {. }
$$

For breeding, male birds are interested in mating with two female birds:

$$
v_{i}=x_{i}+\operatorname{rand} \cdot\left(x_{s, 1}-x_{i}\right)+\operatorname{rand} \cdot\left(x_{s, 2}-x_{i}\right),
$$

where $x_{s, 1}$ and $x_{s, 2}$ are two target vectors which are randomly selected from Type 1 for the interesting mates of the male bird.

\section{The Proposed Population Classification Evolution Algorithm}

In PCE, all individuals in the population are ranked according to their objective function values. The population are divided into two types of evolution based on their rank as follows.

Type 1. The individuals of this type in the population are referred to as elite individuals that have better objective function values than others. The number of these elites $\left(N_{1}\right)$ is determined by (5).

We employ the following expression for the evolution of elite individuals:

$$
v_{i}=x_{i}+(\text { rand }- \text { rand }) \cdot x_{i}
$$

It is worthwhile mentioning that we modify (6) and the disturbance coefficient from (11) is larger than that of (6), which can expand the exploitation space. At the early stage of the optimization process, the larger search step would be faster to search a better solution, which can accelerate convergence.
Type 2. The individuals of this type are referred to as ordinary individuals. The number of these individuals $\left(N_{2}\right)$ is determined by

$$
N_{2}=N P-N_{1}
$$

The DE algorithm [26], which is primarily employed for numerical optimization problems, is a parallel direct search technology that uses NP D-dimensional vectors. In ordinary individuals, we employ the differential evolution " $\mathrm{DE} / \mathrm{best} / 1$ " mutation operator [27] that uses the information of the best individual in the population to guide an individual to be close to the global optimum, which helps to accelerate the convergence speed. The "DE/best/1" operator is as follows:

$$
v_{i}=x_{\mathrm{best}}+\operatorname{rand} \cdot\left(x_{r_{1}}-x_{r_{2}}\right) \text {, }
$$

where indexes $r_{1}$ and $r_{2}$ denote mutually different integers randomly generated from the range $[1, N P] . x_{\text {best }}$ is the bestso-far solution with the best fitness (i.e., lowest objective function value for a minimization problem) in the current population.

Although the "DE/best/1" operator can accelerate the convergence speed, another aspect should be considered. Playing a guiding role only through $x_{\text {best }}$ makes it easier to trap in a local optimum. Thus, a crossover operator following mutation is employed to increase the diversity of the population. The crossover operator is employed to generate the trial vector $u_{i}$ between $x_{i}$ and $v_{i}$ :

$$
u_{i, j}= \begin{cases}v_{i, j}, & \text { if } \text { rand } \leq \text { Cr or } j==r n(i) \\ x_{i, j}, & \text { otherwise }\end{cases}
$$

where $\mathrm{Cr} \in[0,1]$ denotes the crossover rate, which is set from the user; rand $\epsilon[0,1]$ denotes a uniform random number; and $r n(i) \in(1,2, \ldots, D)$ denotes a randomly generated index that insures that $u_{i}$ obtains at least one ingredient from $v_{i}$.

In addition, because Type 3 plays a similar role to Type 2 in SBMO, we remove Type 3; then a random movement of small probability is added to further increase population diversity and strengthen the ability of jumping out of a local optimum for the ordinary evolution [28]. The expression of the random movement is defined as follows:

$$
\begin{aligned}
& \text { for } j=1: D \\
& \qquad v_{i, j}=a_{j}+\text { rand } \cdot\left(b_{j}-a_{j}\right)
\end{aligned}
$$

end,

where $a_{j}$ and $b_{j}$ are the initial lower bound and upper bound of the $j$ th dimension of the $i$ th vector, respectively. 
Step 1 (Initialization)

(1.1) Randomly initialize the entire individuals of population $P=\left\{x_{1}, x_{2}, \ldots, x_{N P}\right\}$ within the upper bound and lower bound;

(1.2) Evaluate fitness of the population $P$ according to the objective function.

Step 2 (The population classification evolution)

Rank the NP individuals according to their fitness, then determine the number of each individual type and classify them, and obtain the best individual

for $i=1: N P$ (all NP individuals in the population)

if individual $i$ th belongs to Type 1

(elite individuals evolution)

Produce the elite individual evolution with (11)

else

(ordinary individuals evolution)

Produce the ordinary individual evolution with (16)

end if

Evaluate whether the evolutionary individual can replace the previous individual using greedy

selection scheme based on the survival of the fittest idea in the nature.

end for

Step 3. If the termination criteria is satisfied, stop; otherwise go to Step 2.

Algorithm 1: Pseudocode of the proposed algorithm.

Therefore, using (13), (14), and (15), the method of ordinary individuals evolution can be defined as follows:

$$
\begin{aligned}
& \text { if rand }<\text { Dep } \\
& v_{i}=x_{\text {best }}+\operatorname{rand} \cdot\left(x_{r_{1}}-x_{r_{2}}\right) \\
& \text { for } j=1: D \\
& \text { if } \text { rand } \leq \mathrm{Cr} \text { or } j==r n(i) \\
& v_{i, j}=v_{i, j} \\
& v_{i, j}=x_{i, j}
\end{aligned}
$$

end if

end for

else

$$
\begin{aligned}
& \text { for } j=1: D \\
& \qquad v_{i, j}=a_{j}+\text { rand } \cdot\left(b_{j}-a_{j}\right)
\end{aligned}
$$

end for

end if,

where Dep $\in[0,1]$ denotes the probability of differential evolution in ordinary individuals. Here, Dep is set to 0.9, which means the probability of the random movement is 0.1 .

An optimization algorithm should be able to satisfactorily compromise between exploitation and exploration to effectively probe the search space [6]. In the proposed population classification evolution approach, the individuals in Type 1 are the neighborhood-based search known as the local search strategy to afford exploitation. Conversely, the other individuals (Type 2) of the population move through the search space with respect to memory and randomness known as the global search to afford exploration. The random movement in Type 2 is utilized to generate a new individual, which may explore a better solution to overcome the premature convergence.

The pseudocode of the proposed PCE algorithm is summarized in Algorithm 1.

\section{Simulation Experiments on Benchmark Functions}

5.1. Experimental Setup. To evaluate the optimal performance of PCE, 13 widely used standard benchmark functions are applied from $[1,29]$. The search space, space dimension, and optimal value of the 13 functions are listed in Table 1. $f_{1}-f_{7}$ belong to unimodal functions, and $f_{8}-f_{13}$ belong to multimodal functions.

The proposed PCE algorithm is compared with six EAs, namely, SBMO [6], CS [30], ABC [31], IADE [17], jDE [29], and GOTLBO [1]. It is worthwhile mentioning that GOTLBO [1], recently proposed by Brest et al., is efficiently utilized to identify the parameters for PV models. Table 2 lists the configuration values of tunable parameters of the mentioned algorithms with reference to the relevant literature.

To simulate the optimization performance among the different algorithms, the performance criteria are employed as follows [1]:

(i) ANFES. ANFES represents the average number of objective function evaluations. It is employed to store the number of objective function evaluations when a solution $x$ satisfying $F(x)-F\left(x^{*}\right) \leq \varepsilon$ is found for each run, where $F\left(x^{*}\right)$ is the best value and $\varepsilon$ is a rather small positive constant as the required accuracy for 
TABLE 1: Benchmark functions.

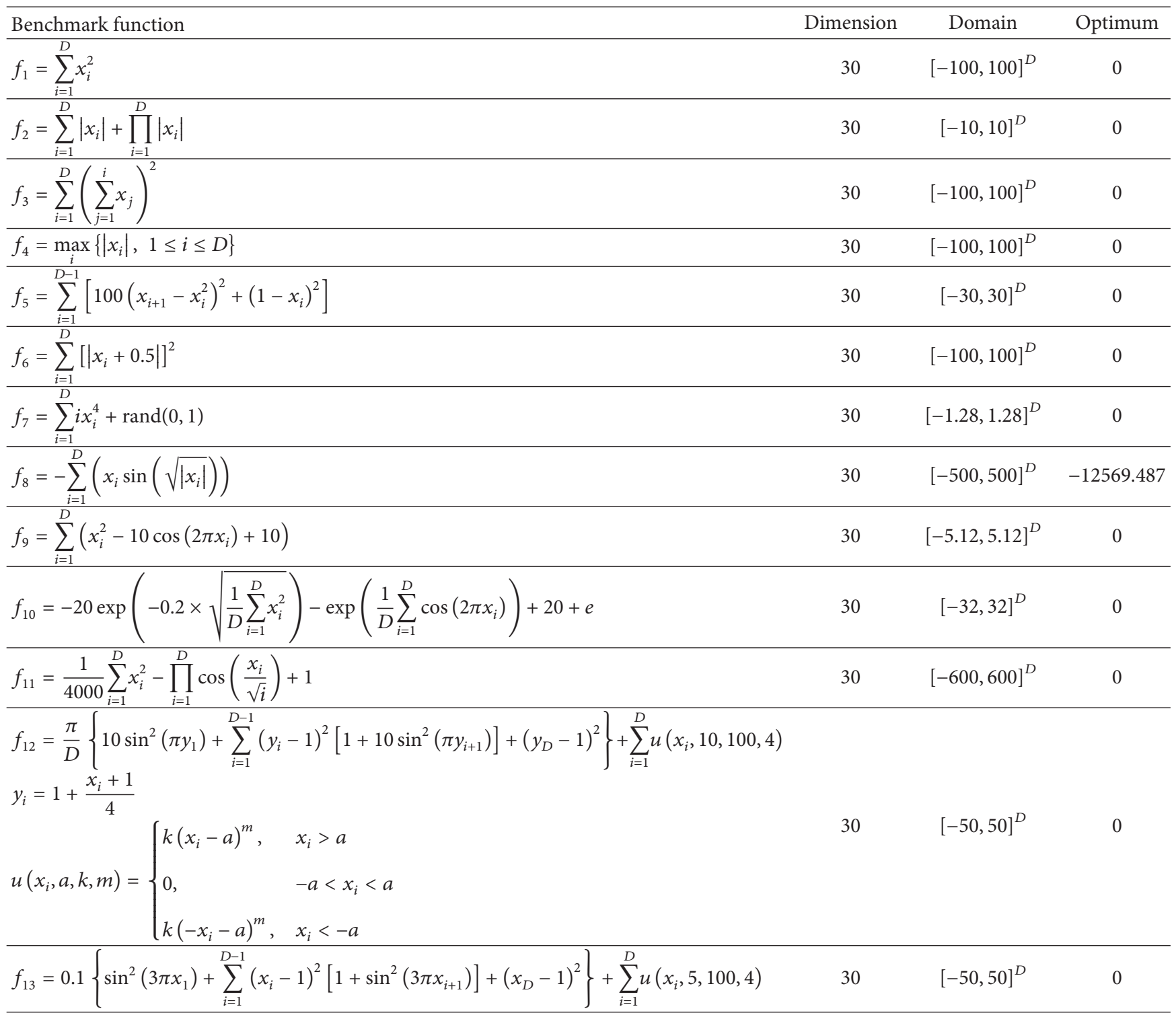

TABle 2: Parameter configuration of seven EAs.

\begin{tabular}{ll}
\hline Algorithm & Parameters \\
\hline SBMO & $N P=30$ \\
CS & $N P=15, P a=0.25$ \\
ABC & $N P=150$ \\
IADE & $N P=100$ \\
jDE & $N P=100, \tau_{1}=\tau_{2}=0.1, F_{l}=0.1, F_{u}=0.9$ \\
GOTLBO & $N P=50, J r=1$ \\
PCE & $N P=100$, Dep $=0.9, \mathrm{Cr}=0.15$ \\
\hline
\end{tabular}

different issues. ANFES can evaluate the convergence speed for an optimization technology.

(ii) SR (Successful Rate). SR represents the number of successful runs among all runs. The successful run signifies that the algorithm can achieve $F(x)-F\left(x^{*}\right) \leq$ $\varepsilon$ before the max. number of objective function evaluations (MaxNFES) condition terminates the optimization process.

Here, the results are obtained in 50 independent runs to ensure a fair comparison for all involved algorithms. For functions $f_{1}(x)-f_{13}(x)$, MaxNFES $=10000 D$, and $\varepsilon=10^{-8}$ $[1,32]$.

5.2. Experimental Results. Table 3 demonstrates the experimental results of seven EAs on 13 standard benchmark functions. Best, Mean, Worst, and Std. signify the best objective function value, mean objective function value, worst objective function value, and standard deviation, respectively. Table 4 shows that six EAs achieve the number of the best performances on 13 benchmark functions.

For the unimodal functions $f_{1}-f_{7}$, PCE yields the optimal results on all unimodal functions. GOTLBO yields the 
TABLE 3: Simulation results for 13 standard benchmark functions.

\begin{tabular}{|c|c|c|c|c|c|}
\hline \multirow{2}{*}{ Benchmark functions } & \multirow{2}{*}{ Methods } & \multicolumn{4}{|c|}{ Results } \\
\hline & & Best & Mean & Worst & Std. \\
\hline \multirow{7}{*}{$f_{1}$} & SBMO & $2.27 E-290$ & $5.77 E-244$ & $2.33 E-242$ & 0 \\
\hline & CS & $1.24 E-57$ & $3.14 E-53$ & $6.14 E-52$ & $9.95 E-53$ \\
\hline & $\mathrm{ABC}$ & $3.67 E-16$ & $6.13 E-16$ & $7.71 E-16$ & $9.38 E-17$ \\
\hline & IADE & $2.55 E-149$ & $1.13 E-145$ & $4.63 E-144$ & $6.53 E-145$ \\
\hline & $\mathrm{jDE}$ & $3.13 E-63$ & $2.46 E-61$ & $5.54 E-60$ & $7.96 E-61$ \\
\hline & GOTLBO & 0 & 0 & 0 & 0 \\
\hline & PCE & 0 & 0 & 0 & 0 \\
\hline \multirow{7}{*}{$f_{2}$} & SBMO & $3.90 E-144$ & $1.65 E-126$ & $8.25 E-125$ & $1.17 E-125$ \\
\hline & CS & $1.21 E-35$ & $5.95 E-33$ & $1.74 E-31$ & $2.48 E-32$ \\
\hline & $\mathrm{ABC}$ & $1.13 E-15$ & $1.50 E-15$ & $1.86 E-15$ & $1.83 E-16$ \\
\hline & IADE & $6.52 E-82$ & $2.41 E-25$ & $1.21 E-23$ & $1.70 E-24$ \\
\hline & $\mathrm{jDE}$ & $2.72 E-37$ & $1.45 E-36$ & $8.37 E-36$ & $1.44 E-36$ \\
\hline & GOTLBO & $1.58 E-288$ & $3.54 E-282$ & $6.89 E-281$ & 0 \\
\hline & PCE & 0 & 0 & 0 & 0 \\
\hline \multirow{7}{*}{$f_{3}$} & SBMO & $3.53 E-297$ & $6.34 E-235$ & $3.01 E-233$ & 0 \\
\hline & CS & $9.39 E-10$ & $1.91 E-07$ & $2.80 E-06$ & $4.40 E-07$ \\
\hline & $\mathrm{ABC}$ & $8.09 E+02$ & $2.28 E+03$ & $4.76 E+03$ & $8.54 E+02$ \\
\hline & IADE & $2.44 E-18$ & $5.82 E+02$ & $3.55 E+03$ & $9.38 E+02$ \\
\hline & jDE & $4.02 E-09$ & $1.43 E-07$ & $6.92 E-07$ & $1.62 E-07$ \\
\hline & GOTLBO & 0 & $9.80 E-306$ & $4.90 E-304$ & 0 \\
\hline & PCE & 0 & 0 & 0 & 0 \\
\hline \multirow{7}{*}{$f_{4}$} & SBMO & $8.09 E-161$ & $1.98 E-144$ & $7.32 E-143$ & $1.06 E-143$ \\
\hline & CS & $8.32 E+00$ & $1.72 E+01$ & $2.81 E+01$ & $4.42 E+00$ \\
\hline & $\mathrm{ABC}$ & $9.04 E+00$ & $1.71 E+01$ & $2.67 E+01$ & $3.49 E+00$ \\
\hline & IADE & $5.02 E-01$ & $6.51 E+00$ & $3.10 E+01$ & $7.22 E+00$ \\
\hline & $\mathrm{jDE}$ & $3.20 E-04$ & $4.67 E-01$ & $3.54 E+00$ & $6.65 E-01$ \\
\hline & GOTLBO & $7.59 E-293$ & $7.64 E-287$ & $1.68 E-285$ & 0 \\
\hline & PCE & 0 & 0 & 0 & 0 \\
\hline \multirow{7}{*}{$f_{5}$} & SBMO & $2.89 E+01$ & $2.90 E+01$ & $2.90 E+01$ & $1.97 E-02$ \\
\hline & CS & $1.33 E-10$ & $4.67 E+00$ & $6.79 E+01$ & $9.93 E+00$ \\
\hline & $\mathrm{ABC}$ & $3.08 E-03$ & $2.57 E-01$ & $3.17 E+00$ & $5.32 E-01$ \\
\hline & IADE & $1.10 E-06$ & $1.96 E+00$ & $1.25 E+01$ & $2.63 E+00$ \\
\hline & $\mathrm{jDE}$ & $7.92 E-01$ & $1.10 E+01$ & $6.72 E+01$ & $1.08 E+01$ \\
\hline & GOTLBO & $6.04 E+00$ & $9.25 E+00$ & $1.45 E+01$ & $1.76 E+00$ \\
\hline & PCE & $9.39 E-29$ & $2.28 E-26$ & $6.52 E-25$ & $9.34 E-26$ \\
\hline \multirow{7}{*}{$f_{6}$} & SBMO & 0 & $1.11 E+01$ & $1.12 E+02$ & $2.15 E+01$ \\
\hline & CS & 0 & $8.14 E-33$ & $7.70 E-32$ & $1.29 E-32$ \\
\hline & $\mathrm{ABC}$ & $4.39 E-16$ & $6.59 E-16$ & $9.59 E-16$ & $1.09 E-16$ \\
\hline & IADE & 0 & $8.00 E-01$ & $5.00 E+00$ & $1.07 E+00$ \\
\hline & $\mathrm{jDE}$ & 0 & 0 & 0 & 0 \\
\hline & GOTLBO & 0 & 0 & 0 & 0 \\
\hline & PCE & 0 & 0 & 0 & 0 \\
\hline \multirow{7}{*}{$f_{7}$} & SBMO & $2.04 E-04$ & $3.82 E-03$ & $1.20 E-02$ & $2.93 E-03$ \\
\hline & CS & $8.77 E-03$ & $3.92 E-02$ & $1.45 E-01$ & $2.44 E-02$ \\
\hline & $\mathrm{ABC}$ & $7.99 E-02$ & $1.68 E-01$ & $2.34 E-01$ & $3.36 E-02$ \\
\hline & IADE & $7.26 E-04$ & $2.21 E-03$ & $4.97 E-03$ & $9.58 E-04$ \\
\hline & jDE & $1.01 E-03$ & $3.23 E-03$ & $4.70 E-03$ & $8.35 E-04$ \\
\hline & GOTLBO & $3.26 E-05$ & $9.16 E-05$ & $2.09 E-04$ & $4.24 E-05$ \\
\hline & PCE & $1.43 E-06$ & $2.76 E-05$ & $8.09 E-05$ & $2.03 E-05$ \\
\hline
\end{tabular}


TABle 3: Continued.

\begin{tabular}{|c|c|c|c|c|c|}
\hline \multirow{2}{*}{ Benchmark functions } & \multirow{2}{*}{ Methods } & \multicolumn{4}{|c|}{ Results } \\
\hline & & Best & Mean & Worst & Std. \\
\hline \multirow{7}{*}{$f_{8}$} & SBMO & -12539.38532 & -8088.03669 & -64992.35445 & $9.30 E+03$ \\
\hline & CS & -12569.48661 & -12046.97452 & -11306.10604 & $2.52 E+02$ \\
\hline & $\mathrm{ABC}$ & -12569.48662 & -12569.48662 & -12569.48661 & $1.28 E-06$ \\
\hline & IADE & -12075.83083 & -11118.32618 & -9598.627861 & $5.53 E+02$ \\
\hline & jDE & -12569.48662 & -12569.48662 & -12569.48662 & $7.35 E-12$ \\
\hline & GOTLBO & -11462.53150 & -9343.52209 & -7871.30159 & $7.02 E+02$ \\
\hline & PCE & -12569.48662 & -12569.48662 & -12569.48662 & $2.80 E-12$ \\
\hline \multirow{7}{*}{$f_{9}$} & SBMO & $1.06 E+02$ & $1.58 E+02$ & $2.00 E+01$ & $2.08 E+01$ \\
\hline & CS & $9.95 E-01$ & $7.92 E+01$ & $1.99 E+01$ & $4.16 E+01$ \\
\hline & $\mathrm{ABC}$ & $5.68 E-14$ & $9.70 E-13$ & $2.28 E-11$ & $3.22 E-12$ \\
\hline & IADE & $1.90 E+01$ & $3.71 E+01$ & $7.44 E+01$ & $1.04 E+01$ \\
\hline & jDE & 0 & 0 & 0 & 0 \\
\hline & GOTLBO & 0 & 0 & 0 & 0 \\
\hline & PCE & 0 & 0 & 0 & 0 \\
\hline \multirow{7}{*}{$f_{10}$} & SBMO & 0 & $5.11 E-01$ & $8.00 E+00$ & $2.04 E+00$ \\
\hline & CS & $9.31 E-01$ & $1.89 E+00$ & $3.93 E+00$ & $6.60 E-01$ \\
\hline & $\mathrm{ABC}$ & $4.35 E-14$ & $5.52 E-14$ & $6.48 E-14$ & $6.26 E-15$ \\
\hline & IADE & $7.11 E-15$ & $1.22 E-01$ & $1.50 E+00$ & $3.74 E-01$ \\
\hline & jDE & $3.55 E-15$ & $4.05 E-15$ & $7.11 E-15$ & $1.25 E-15$ \\
\hline & GOTLBO & 0 & $2.70 E-15$ & $3.55 E-15$ & $1.53 E-15$ \\
\hline & PCE & 0 & 0 & 0 & 0 \\
\hline \multirow{7}{*}{$f_{11}$} & SBMO & 0 & $1.18 E-01$ & $1.03 E+00$ & $3.24 E-01$ \\
\hline & CS & 0 & $5.12 E-03$ & $5.64 E-03$ & $1.07 E-02$ \\
\hline & $\mathrm{ABC}$ & $1.11 E-16$ & $3.06 E-14$ & $4.84 E-13$ & $7.69 E-14$ \\
\hline & IADE & 0 & $8.45 E-03$ & $5.86 E-02$ & $1.24 E-03$ \\
\hline & jDE & 0 & 0 & 0 & 0 \\
\hline & GOTLBO & 0 & 0 & 0 & 0 \\
\hline & PCE & 0 & 0 & 0 & 0 \\
\hline \multirow{7}{*}{$f_{12}$} & SBMO & $9.41 E-01$ & $9.39 E+00$ & $1.92 E+01$ & $5.22 E+00$ \\
\hline & CS & $1.57 E-32$ & $2.28 E-01$ & $2.76 E+00$ & $5.66 E-01$ \\
\hline & $\mathrm{ABC}$ & $4.53 E-16$ & $6.01 E-16$ & $7.74 E-16$ & $9.09 E-17$ \\
\hline & IADE & $1.57 E-32$ & $8.77 E-02$ & $1.56 E+00$ & $2.56 E-01$ \\
\hline & jDE & $1.57 E-32$ & $1.57 E-32$ & $1.57 E-32$ & $1.55 E-29$ \\
\hline & GOTLBO & $2.09 E-32$ & $3.69 E-31$ & $1.65 E-30$ & $5.80 E-31$ \\
\hline & PCE & $3.12 E-32$ & $3.23 E-30$ & $1.04 E-28$ & $1.55 E-29$ \\
\hline \multirow{7}{*}{$f_{13}$} & SBMO & $3.00 E-02$ & $1.25 E+01$ & $4.16 E+01$ & $1.17 E+01$ \\
\hline & CS & $1.35 E-32$ & $3.68 E-01$ & $8.06 E+00$ & $1.29 E+00$ \\
\hline & $\mathrm{ABC}$ & $4.52 E-16$ & $6.74 E-16$ & $9.31 E-16$ & $1.08 E-16$ \\
\hline & IADE & $1.35 E-32$ & $4.93 E-03$ & $1.06 E-01$ & $1.57 E-02$ \\
\hline & jDE & $1.35 E-32$ & $1.35 E-32$ & $1.35 E-32$ & $1.11 E-47$ \\
\hline & GOTLBO & $2.21 E-32$ & $3.84 E-02$ & $3.48 E-01$ & $5.76 E-02$ \\
\hline & PCE & $1.35 E-32$ & $7.21 E-32$ & $2.16 E-30$ & $3.02 E-31$ \\
\hline
\end{tabular}

Italics highlight the best performance.

optimal results on 2 functions. jDE yields the optimal result on 1 function. The other four EAs do not obtain the optimal performance.

For the multimodal functions $f_{8}-f_{13}$, PCE obtains the optimal result on 4 functions, $f_{8}-f_{11}$. jDE also obtains the optimal results on 4 functions, $f_{9}$ and $f_{11}-f_{13}$. GOTLBO obtains the optimal results on 2 functions, $f_{9}$ and $f_{11}$, whereas the other four EAs do not obtain the optimal performance.

In general, on 13 benchmark functions, PCE is superior to the other six EAs, obtaining the best performance on 11 functions, $f_{1}-f_{11}$. 
TABLE 4: The number of the optimal performances on 13 standard benchmark functions.

\begin{tabular}{lccccccc}
\hline & SBMO & CS & ABC & IADE & jDE & GOTLBO & PCE \\
\hline$f_{1}-f_{7}$ & 0 & 0 & 0 & 0 & 1 & 2 & 7 \\
$f_{8}-f_{13}$ & 0 & 0 & 0 & 0 & 4 & 2 & 4 \\
\hline Total & 0 & 0 & 0 & 0 & 5 & 4 & 11 \\
\hline
\end{tabular}

TABLE 5: The SR and ANFES results.

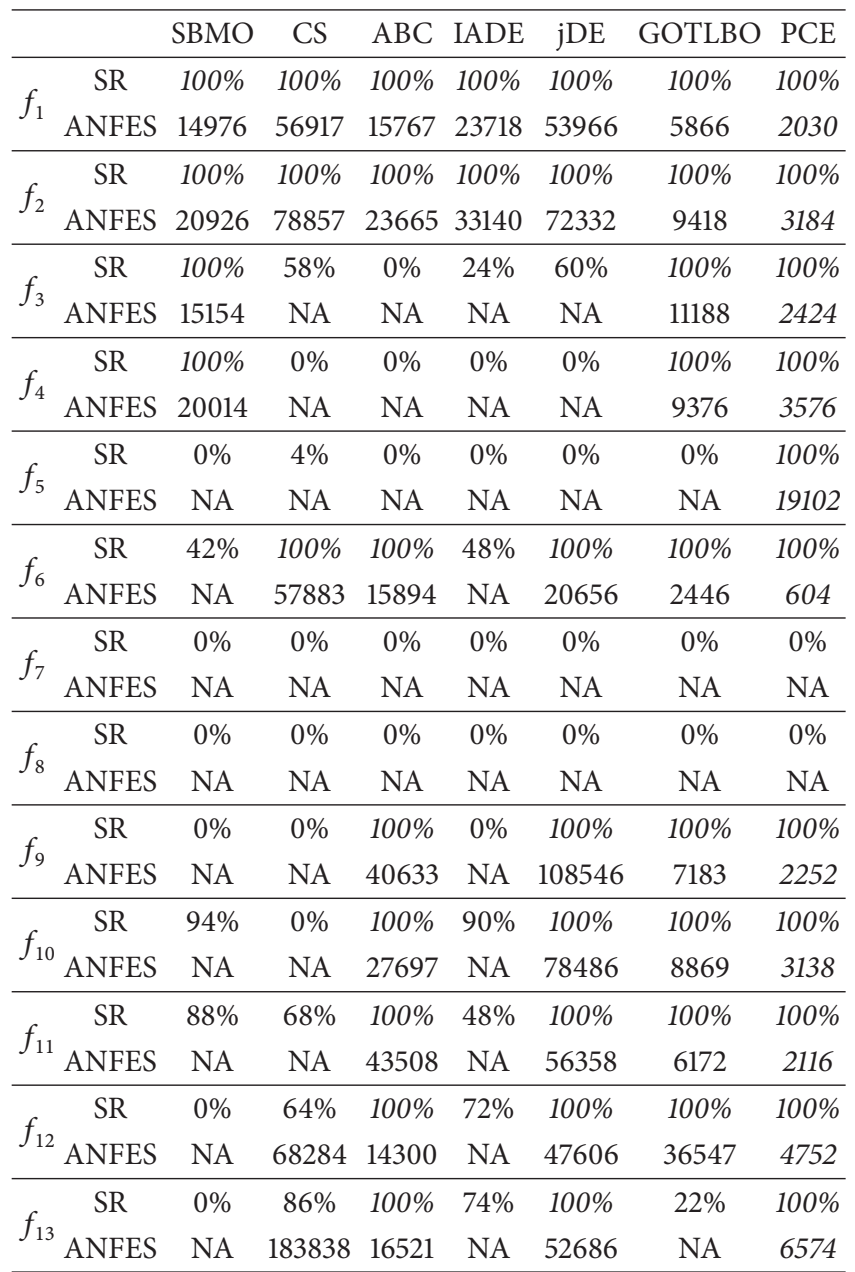

Note. NA implies that $F(x)-F\left(x^{*}\right) \leq \varepsilon$ is not achieved until MaxNFES. Italics highlight the best performance.

The SR and ANFES are listed in Table 5. For the unimodal functions $f_{1}-f_{7}$, PCE obtains optimal results compared to the other six EAs for the optimal SR on 6 functions $f_{1}-f_{6}$. For function $f_{5}$, only the SR of PCE can reach $100 \%$. GOTLBO obtains the optimal SR on 5 functions, $f_{1}-f_{4}$ and $f_{6}$. CS, ABC, and jDE obtain the optimal SR on 3 functions, $f_{1}-f_{2}$ and $f_{6}$. SBMO obtains the optimal SR on 4 functions, $f_{1}-f_{4}$. IADE obtains the optimal SR on 2 functions, $f_{1}-f_{2}$. For the ANFES, PCE exhibits a faster convergence speed than the other six EAs on 6 functions, $f_{1}-f_{6}$.

For the multimodal functions $f_{8}-f_{13}, \mathrm{PCE}, \mathrm{ABC}$, and jDE yield the optimal SR on 5 functions. GOTLBO obtains the optimal SR on 4 functions. For the ANFES values, PCE obtains the best results among seven EAs on 5 functions, whereas the other six EAs do not obtain the optimal ANFES.

5.3. Discussion. The common benchmark functions have been applied to estimate the optimization performance of PCE compared with six representative EAs. According to the experimental results, we can summarize the following:

(i) PCE has a faster convergence speed than SBMO, CS, $A B C$, IADE, jDE, and GOTLBO on all unimodal functions. Additionally, PCE obtains the optimal performance on all unimodal functions when comparing it with these six EAs.

(ii) On most multimodal functions, PCE has a faster convergence speed and higher accuracy than SBMO, $\mathrm{CS}, \mathrm{ABC}$, IADE, jDE, and GOTLBO.

(iii) PCE is especially suitable to be used on unimodal functions.

On the whole, the proposed PCE algorithm has the best performance among all compared algorithms.

\section{Parameter Extraction for Solar Cell Models Using PCE}

The $I-V$ characteristics of a commercial silicon solar cell (RTC France) with $57 \mathrm{~mm}$ diameter are employed to test the performance of PCE for parameter extraction technology. The real data have been employed under 1 sun $\left(1000 \mathrm{~W} / \mathrm{m}^{2}\right)$ at $33^{\circ} \mathrm{C}[1,8]$. The $(I-V)$ characteristics of the extracted parameters by PCE are compared with the $(I-V)$ characteristics which are obtained from [8]. The $I-V$ data are listed in Table 6. For the single and double diode models, the search spaces of each parameter are listed in Table $7[1,4]$.

6.1. Comparison with Other EAs. In this subsection, PCE is compared with six EAs for solar cell models to show its optimization performance.

For a single diode model, MaxNFES is 10,000 , whereas, for a double diode model, MaxNFES is 20,000. The constant $\varepsilon$ is set to be 0.002 for both the single diode model and the double diode model [1]. The configuration values of tunable parameters of the mentioned algorithms with reference to the relevant literature are presented in Table 8 , and every algorithm is independently run 50 times.

Table 9 shows the comparative results for the single diode model. PCE obtains best results among the best, mean, worst, and Std. RMSE values. jDE obtains the best RMSE value the same as PCE with a value of $9.86022 E-04$. For the SR value, CS, jDE, GOTLBO, and PCE achieve a value of $100 \%$. PCE obtains best value of 1380 in terms of ANFES. Obviously, PCE has a more stable performance compared with other six algorithms for the single diode model based on RMSE, SR, and ANFES.

For a single diode model, the convergence graph of different EAs is plotted in Figure 3. Figure 3 shows that in the whole stage PCE converges the fastest, followed by jDE. PCE is capable of successively converging toward the optimal solutions during the whole evolutionary process. 
TABLE 6: The $I-V$ data of the solar cell, RTC France.

\begin{tabular}{|c|c|c|}
\hline & $V_{t}(\mathrm{~V})$ & $I_{t}(\mathrm{~A})$ \\
\hline 1 & -0.2057 & 0.764 \\
\hline 2 & -0.1291 & 0.762 \\
\hline 3 & -0.0588 & 0.7605 \\
\hline 4 & 0.0057 & 0.7605 \\
\hline 5 & 0.0646 & 0.76 \\
\hline 6 & 0.1185 & 0.759 \\
\hline 7 & 0.1678 & 0.757 \\
\hline 8 & 0.2132 & 0.757 \\
\hline 9 & 0.2545 & 0.7555 \\
\hline 10 & 0.2924 & 0.754 \\
\hline 11 & 0.3269 & 0.7505 \\
\hline 12 & 0.3585 & 0.7465 \\
\hline 13 & 0.3873 & 0.7385 \\
\hline 14 & 0.4137 & 0.728 \\
\hline 15 & 0.4373 & 0.7065 \\
\hline 16 & 0.459 & 0.6755 \\
\hline 17 & 0.4784 & 0.632 \\
\hline 18 & 0.496 & 0.573 \\
\hline 19 & 0.5119 & 0.499 \\
\hline 20 & 0.5265 & 0.413 \\
\hline 21 & 0.5398 & 0.3165 \\
\hline 22 & 0.5521 & 0.212 \\
\hline 23 & 0.5633 & 0.1035 \\
\hline 24 & 0.5736 & -0.01 \\
\hline 25 & 0.5833 & -0.123 \\
\hline 26 & 0.59 & -0.21 \\
\hline
\end{tabular}

TABLE 7: The search spaces for a single diode model and double diode model.

\begin{tabular}{lcc}
\hline Parameter & Lower bound & Upper bound \\
\hline$I_{\mathrm{ph}}(\mathrm{A})$ & 0 & 1 \\
$I_{\mathrm{sd}}, I_{\mathrm{sd} 1}, I_{\mathrm{sd} 2}(\mu \mathrm{A})$ & 0 & 1 \\
$R_{\mathrm{s}}(\Omega)$ & 0 & 0.5 \\
$R_{\mathrm{sh}}(\Omega)$ & 0 & 100 \\
$n, n_{1}, n_{2}$ & 1 & 2 \\
\hline
\end{tabular}

TABLE 8: Parameter settings of seven EAs.

\begin{tabular}{ll}
\hline Algorithm & Parameters \\
\hline SBMO & $N P=30$ \\
CS & $N P=15, P a=0.25$ \\
ABC & $N P=150$ \\
IADE & $N P=100$ \\
jDE & $N P=20, \tau_{1}=\tau_{2}=0.1, F_{l}=0.1, F_{u}=0.9$ \\
GOTLBO & $N P=20, J r=0.4$ \\
PCE & $N P=100$, Dep $=0.9, \mathrm{Cr}=0.15$ \\
\hline
\end{tabular}

For the double diode model, the comparative data are shown in Table 10. PCE obtains best results among the best, mean, worst, and Std. RMSE values, SR, and ANFES

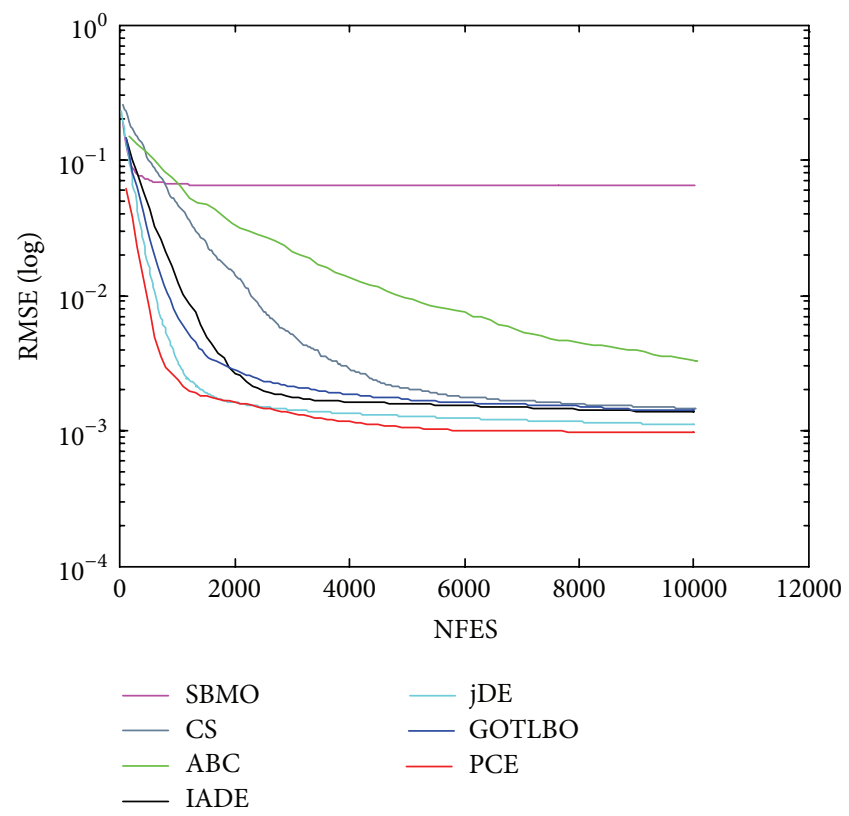

FIgURE 3: The convergence graph of different EAs for the single diode model.

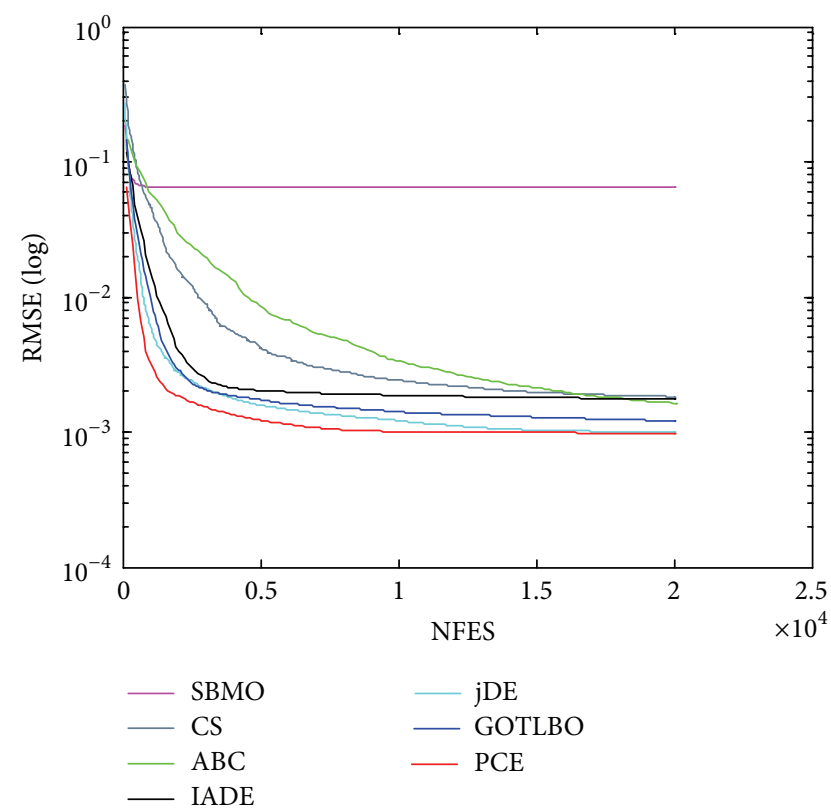

FIGURE 4: The convergence graph of different EAs for the double diode model.

compared with the other six EAs, which means PCE is superior to other six algorithms.

For a single diode model, the convergence graph of different EAs is plotted in Figure 4. Figure 4 shows that in the whole stage PCE converges the fastest. Therefore, PCE is capable of successively converging toward the optimal solutions during the whole evolutionary process.

6.2. Compared with the Results of the Literature. In this

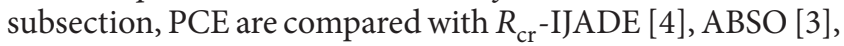


TABLE 9: Simulation results for the single diode model.

\begin{tabular}{|c|c|c|c|c|c|c|c|}
\hline \multirow{2}{*}{ Algorithm } & \multicolumn{4}{|c|}{ RMSE } & \multirow{2}{*}{ SR } & \multicolumn{2}{|c|}{ ANFES } \\
\hline & Best & Mean & Worst & Std. & & Mean & Std. \\
\hline SBMO & $1.03062 E-03$ & $3.88890 E-03$ & $4.74555 E-02$ & $7.14069 E-03$ & $44 \%$ & NA & NA \\
\hline CS & $1.02642 E-03$ & $1.46928 E-03$ & $1.94019 E-03$ & $2.42769 E-04$ & $100 \%$ & 5859 & 1407 \\
\hline $\mathrm{ABC}$ & $1.48749 E-03$ & $3.31301 E-03$ & $7.75872 E-03$ & $1.10844 E-03$ & $8 \%$ & NA & NA \\
\hline IADE & $9.86068 E-04$ & $1.37137 E-03$ & $3.75573 E-03$ & $5.42916 E-04$ & $92 \%$ & NA & NA \\
\hline jDE & $9.86022 E-04$ & $1.10575 E-03$ & $1.54390 E-03$ & $1.37055 E-04$ & $100 \%$ & 1734 & 822 \\
\hline GOTLBO & $9.86179 E-04$ & $1.30270 E-03$ & $1.98800 E-03$ & $2.75957 E-04$ & $100 \%$ & 3438 & 2325 \\
\hline PCE & $9.86022 E-04$ & $9.86022 E-04$ & $9.86022 E-04$ & $3.05726 E-12$ & $100 \%$ & 1380 & 661 \\
\hline
\end{tabular}

Note. NA implies that $F(x)-F\left(x^{*}\right) \leq \varepsilon$ is not achieved until MaxNFES. Italics highlight the best performance.

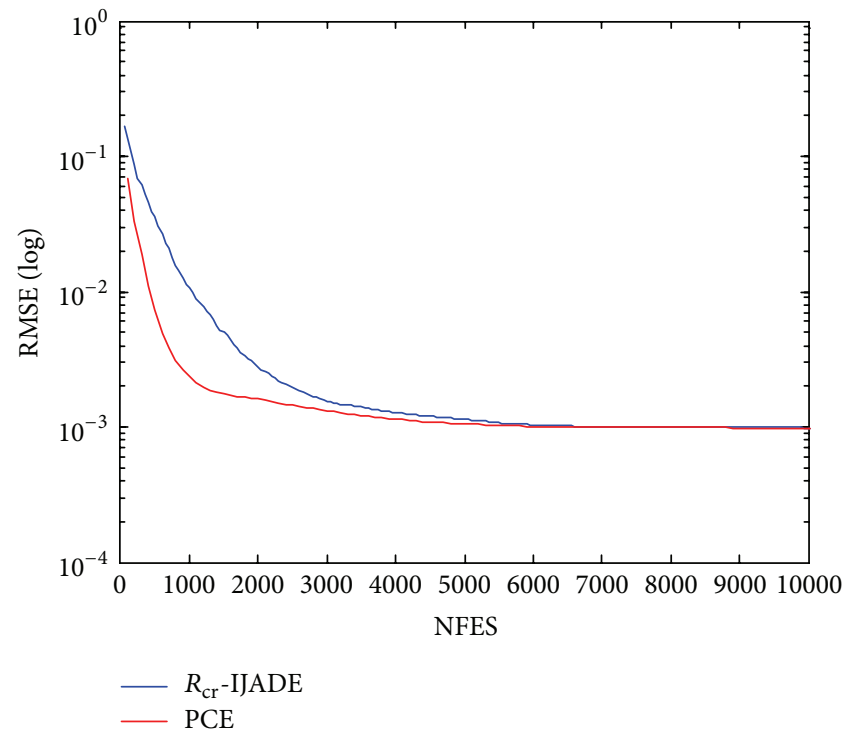

(a)

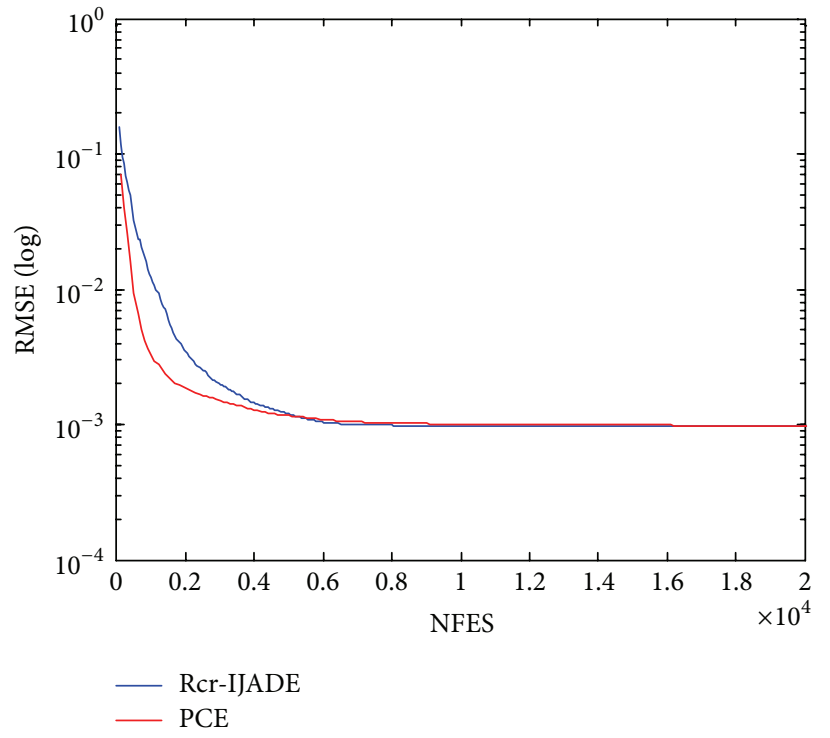

(b)

FIGURE 5: The convergence graphs of different EAs for (a) the single diode model and (b) the double diode model.

IGHS [21], and GOTLBO [1], in terms of the five parameters for the single diode model or the seven parameters for the double diode model. Additionally, the optimal RMSE value is also compared from the related literature. These algorithms are adopted for comparison because of their good performance.

Table 11 records the comparative results for a single diode model. PCE and $R_{\mathrm{cr}}$-IJADE achieve the optimal RMSE value that is $9.86022 E-04$, which is better than other compared algorithms. The RMSE values from good to bad for GOTLBO, ABSO, and IGHS are 9.87442E - 04, 9.9124E - 04, and $9.9306 E-04$, respectively. The computational cost of an optimization algorithm adopts the NFES to characterization. NFES in the last row of Table 11 is the number of objective function evaluations for one run [1]. The NFES for PCE, $R_{\mathrm{cr}}{ }^{-}$ IJADE, and GOTLBO are set to be 10,000, whereas the NFES for ABSO and IGHS are set to be 150,000.

For the double diode model, the comparative results are recorded in Table 12. PCE and $R_{\mathrm{cr}}$-IJADE achieve the optimal RMSE value, that is, $9.8248 E-04$, which is better than other compared algorithms. GOTLBO obtains the second best RMSE value $(9.83117 E-04)$, which is rather close to the RMSE value of PCE and $R_{c r}$-IJADE. The RMSE values from good to bad for ABSO and IGHS are 9.8344E - 04 and $9.8635 E-04$, respectively. The NFES for PCE, $R_{\mathrm{cr}}-\mathrm{IJADE}$, and GOTLBO are set to be 20,000, whereas the NFES for ABSO and IGHS are set to be 150,000 .

According to Tables 11 and 12, it is clear that the results of PCE are similar to those of $R_{\mathrm{cr}}$-IJADE. Therefore, the convergence graphs of both PCE and $R_{\mathrm{cr}}$-IJADE that are employed to further demonstrate the optimization performance are plotted in Figure 5. The convergence graph for the single diode model shows that PCE has a faster convergence speed than $R_{\mathrm{cr}}$-IJADE. Since about 6700 NFEs, the convergence of PCE and $R_{\mathrm{cr}}$-IJADE is almost consistent. The convergence graph for the double diode model shows that in the early stage PCE has faster convergence. Later, the convergence speeds of both PCE and $R_{\mathrm{cr}}$-IJADE are very close and tend to be of a similar value. Therefore, PCE has a faster convergence speed than $R_{\mathrm{cr}}$-IJADE for both the single and double diode model. 


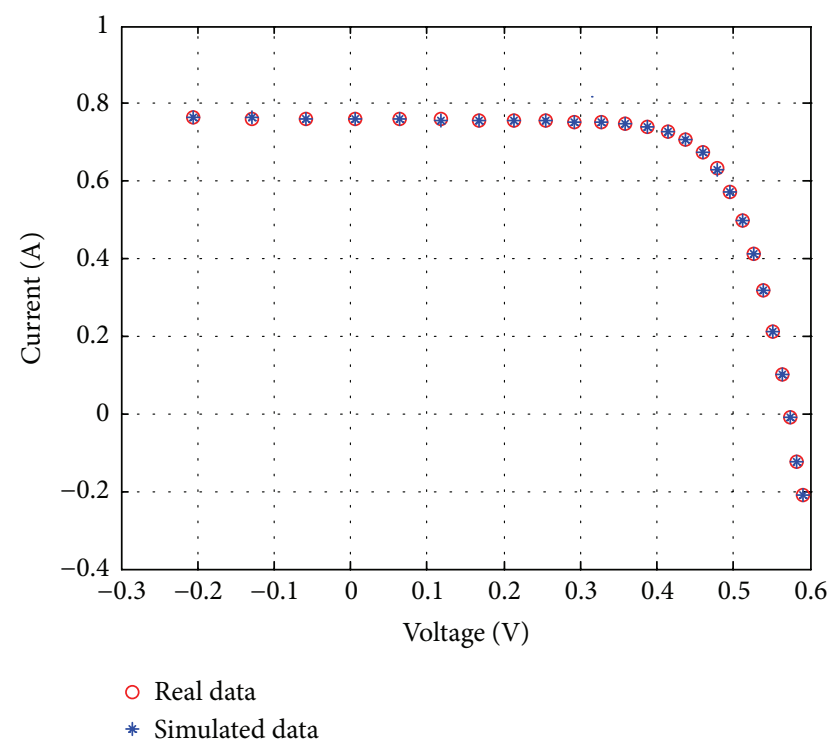

(a)

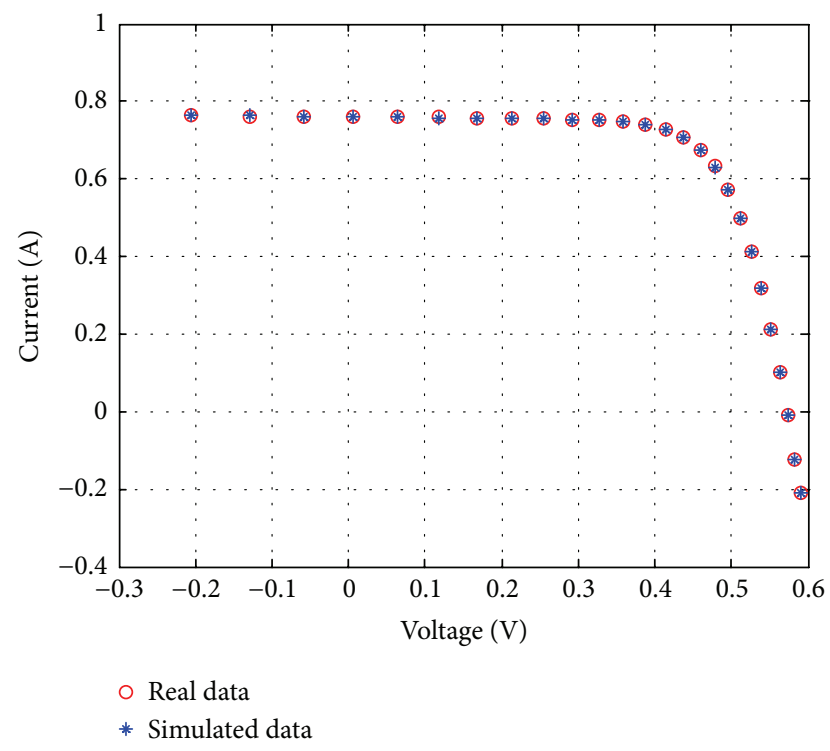

(b)

FIGURE 6: Comparison results from the real data and simulated data obtained by PCE for (a) the single diode model and (b) the double diode model.

TABLE 10: Simulation results for the double diode model.

\begin{tabular}{|c|c|c|c|c|c|c|c|}
\hline \multirow{2}{*}{ Algorithm } & \multicolumn{4}{|c|}{ RMSE } & \multirow{2}{*}{ SR } & \multicolumn{2}{|c|}{ ANFES } \\
\hline & Best & Mean & Worst & Std. & & Mean & Std. \\
\hline SBMO & $1.11833 E-03$ & $3.90818 E-03$ & $1.60974 E-02$ & $2.99964 E-03$ & $22 \%$ & NA & NA \\
\hline CS & $1.05191 E-03$ & $1.72124 E-03$ & $2.67872 E-03$ & $4.43650 E-04$ & $74 \%$ & NA & NA \\
\hline $\mathrm{ABC}$ & $1.00024 E-03$ & $1.61831 E-03$ & $2.55927 E-03$ & $3.34299 E-04$ & $88 \%$ & NA & NA \\
\hline IADE & $9.83214 E-04$ & $1.52217 E-03$ & $3.73289 E-03$ & $5.68918 E-04$ & $86 \%$ & NA & NA \\
\hline jDE & $9.83021 E-04$ & $1.03218 E-03$ & $1.03218 E-03$ & $1.47210 E-04$ & $100 \%$ & 4390 & 2873 \\
\hline GOTLBO & $9.85986 E-04$ & $1.24102 E-03$ & $1.95115 E-03$ & $2.39333 E-04$ & $100 \%$ & 5416 & 3914 \\
\hline PCE & $9.82483 E-04$ & $9.86100 E-04$ & $1.02516 E-03$ & $5.99505 E-06$ & $100 \%$ & 1860 & 1105 \\
\hline
\end{tabular}

Note. NA implies that $F(x)-F\left(x^{*}\right) \leq \varepsilon$ is not achieved until MaxNFES.

Italics highlight the best performance.

Figure 6 shows the $I-V$ behaviors obtained by PCE along with the real data for the single diode model and double diode model. The results explicitly manifest the simulated data obtained by PCE as being highly consistent with the real data, which indicates that the identified parameters with PCE are rather precise.

6.3. Results for PCE Tested with an Experimental Data from the Manufacturer's Data Sheet. Here the proposed PCE technique is employed to extract the optimal parameters of the single diode model for three solar modules of different types (monocrystalline (SM55) [33], thin-film (ST40) [34], and multicrystalline (KC200GT) [35]). The $(I-V)$ characteristics of the extracted parameters by PCE are compared with the $(I-V)$ characteristics which are obtained from the manufacturer's data sheets for the same modules and at the same environmental conditions. The real data was collected at five different irradiance levels of mainly $1000 \mathrm{~W} / \mathrm{m}^{2}, 800 \mathrm{~W} / \mathrm{m}^{2}, 600 \mathrm{~W} / \mathrm{m}^{2}, 400 \mathrm{~W} / \mathrm{m}^{2}$, and
$200 \mathrm{~W} / \mathrm{m}^{2}$, with constant temperature and at three different levels of temperatures, with constant irradiance.

The optimal parameters are extracted and illustrated in Tables $13-15$ of the single diode model for the three types of the solar modules at different temperatures. Moreover, Table 16 is provided for the optimal extracted parameters at different levels of irradiance. The $(I-V)$ characteristics are plotted for the three solar modules at different temperatures as shown in Figures 7-9 and at different irradiance levels as shown in Figures 10-12.

It can be observed that the estimated optimal parameters by PCE technique indicate a more accurate $(I-V)$ curves over the entire ranges of the real data set with very low RMSE at all irradiance levels and temperature values under test. This also shows the good performance of the proposed PCE technique. Moreover, the new PCE technique achieves accurate extraction of the solar modules parameters at low irradiance which is very crucial when the module is subjected to certain mismatch conditions such as partial shading. 
TABLE 11: Data comparison of the single diode model from the related literature.

\begin{tabular}{|c|c|c|c|c|c|}
\hline Algorithm & IGHS & ABSO & $R_{\mathrm{cr}}$-IJADE & GOTLBO & PCE \\
\hline$I_{\mathrm{ph}}(\mathrm{A})$ & 0.7608 & 0.7608 & 0.760776 & 0.760789 & 0.760776 \\
\hline$I_{\mathrm{sd}}(\mu \mathrm{A})$ & 0.3435 & 0.360623 & 0.323921 & 0.324506 & 0.323021 \\
\hline$R_{s}(\Omega)$ & 0.03659 & 0.03659 & 0.036377 & 0.036358 & 0.036377 \\
\hline$R_{\mathrm{sh}}(\Omega)$ & 53.2845 & 52.2903 & 53.718526 & 53.631998 & 53.718525 \\
\hline$n$ & 1.4874 & 1.47878 & 1.481184 & 1.481538 & 1.481074 \\
\hline RMSE & $9.9306 E-04$ & $9.9124 E-04$ & $9.86022 E-04$ & $9.87442 E-04$ & $9.86022 E-04$ \\
\hline NFES & 150,000 & 150,000 & 10,000 & 10,000 & 10,000 \\
\hline
\end{tabular}

Italics highlight the best performance.

TABLE 12: Data comparison of the double diode model from the related literature.

\begin{tabular}{|c|c|c|c|c|c|}
\hline Algorithm & IGHS & ABSO & $R_{\mathrm{cr}}$-IJADE & GOTLBO & PCE \\
\hline$I_{\mathrm{ph}}(\mathrm{A})$ & 0.7608 & 0.76077 & 0.760781 & 0.760810 & 0.760781 \\
\hline$I_{\mathrm{sd} 1}(\mu \mathrm{A})$ & 0.9731 & 0.26713 & 0.225974 & 0.166249 & 0.226015 \\
\hline$R_{s}(\Omega)$ & 0.0369 & 0.03657 & 0.03674 & 0.036952 & 0.03674 \\
\hline$R_{\mathrm{sh}}(\Omega)$ & 53.8368 & 54.6219 & 55.485443 & 55.158863 & 55.483160 \\
\hline$n_{1}$ & 1.9213 & 1.46512 & 1.451017 & 1.427801 & 1.450923 \\
\hline$I_{\mathrm{sd} 2}(\mu \mathrm{A})$ & 0.1679 & 0.38191 & 0.749347 & 0.738203 & 0.749340 \\
\hline$n_{2}$ & 1.4281 & 1.98152 & 2 & 1.865679 & 2 \\
\hline RMSE & $9.8635 E-04$ & $9.8344 E-04$ & $9.8248 E-04$ & $9.83117 E-04$ & $9.8248 E-04$ \\
\hline NFES & 150,000 & 150,000 & 20,000 & 20,000 & 20,000 \\
\hline
\end{tabular}

Italics highlight the best performance.

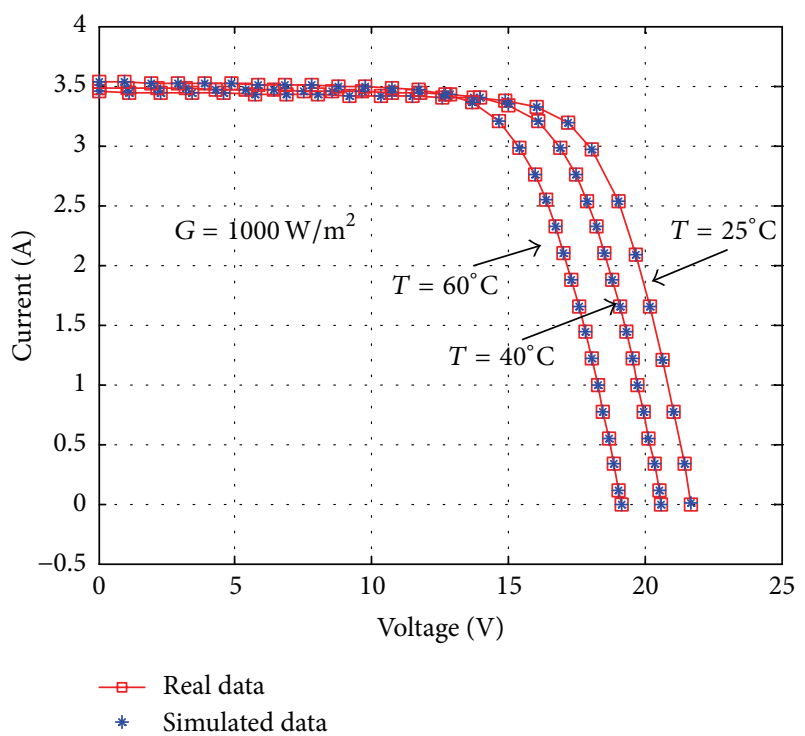

Figure 7: Comparison between real data and the simulated data by PCE for monocrystalline SM55 at different temperatures.

\section{Conclusions}

In this paper, inspired by the mating strategies, we develop a novel optimization method referred to as PCE to overcome the slow convergence of SBMO. In PCE, there are three adjustable parameters: NP (all individuals in the population), Dep (the differential evolution probability), and $\mathrm{Cr}$ (the

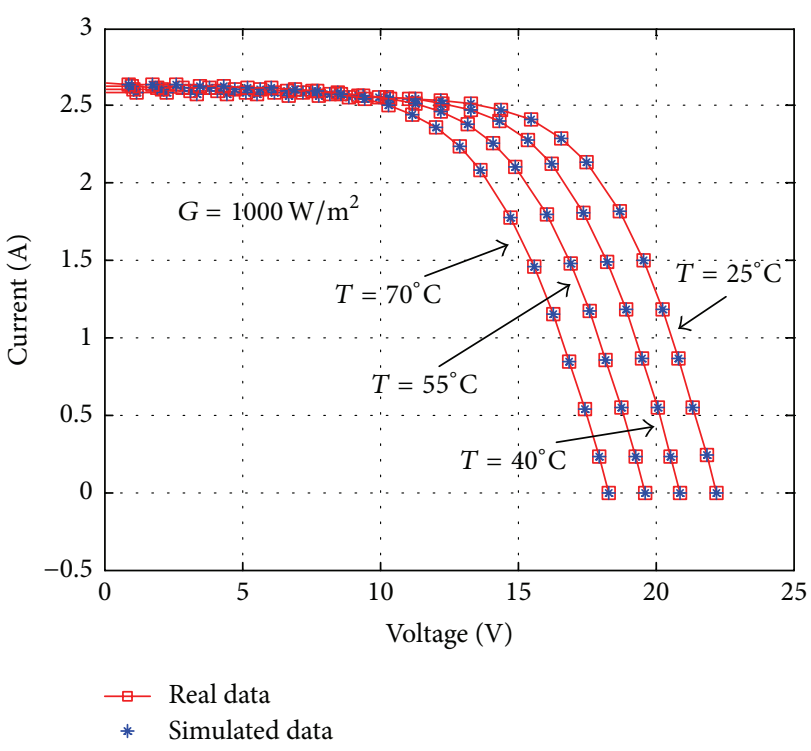

FIGURE 8: Comparison between real data and the simulated data by PCE for thin-film ST40 at different temperatures.

crossover rate). In this study, the parameters are tuned to $N P=100$, Dep $=0.9$, and $\mathrm{Cr}=0.15$.

To verify the optimization performance of PCE, we compare it with six EAs, that is, SBMO, CS, ABC, IADE, jDE, and GOTLBO. PCE is initially estimated on 13 classic benchmark functions. The simulated data indicate that PCE 


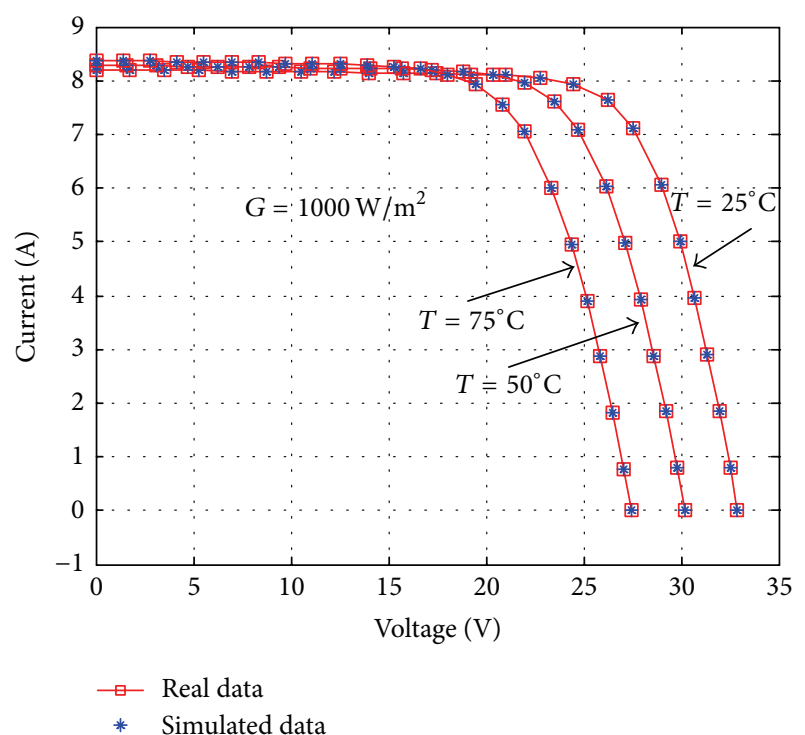

Figure 9: Comparison between real data and the simulated data by PCE for multicrystalline KC200GT at different temperatures.

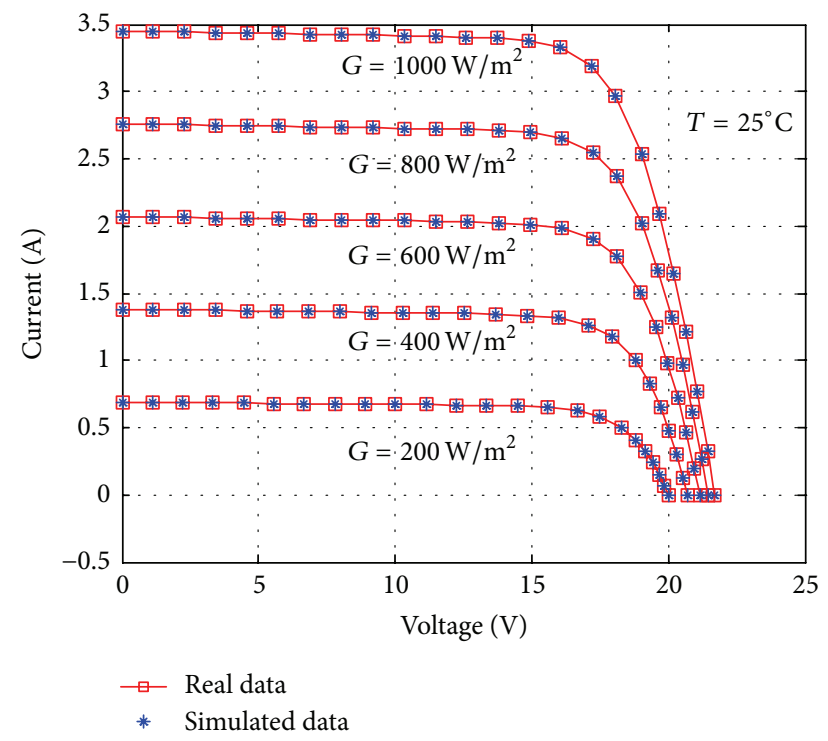

FIGURE 10: Comparison between real data and the simulated data by PCE for monocrystalline SM55 at different irradiances.

has a faster convergence speed and higher accuracy, and it has the best results on 11 functions among these six algorithms.

Then, PCE is applied to accurately estimate the parameter of solar cell models like the single and double diode model. PCE performs the best performance among the six algorithms. For the single diode model, PCE yields the best RMSE value of $9.86022 E-04$. For the double diode model, PCE yields the best RMSE value of $9.8248 E-04$. Compared with other optimization algorithms in the literature, such as IGHS, ABSO, $R_{\mathrm{cr}}$-IJADE, and GOTLBO, the final convergence of PCE and $R_{\mathrm{cr}}$-IJADE is almost consistent for both the single and double diode model. However, the convergence graphs for both the single and double diode models show that in

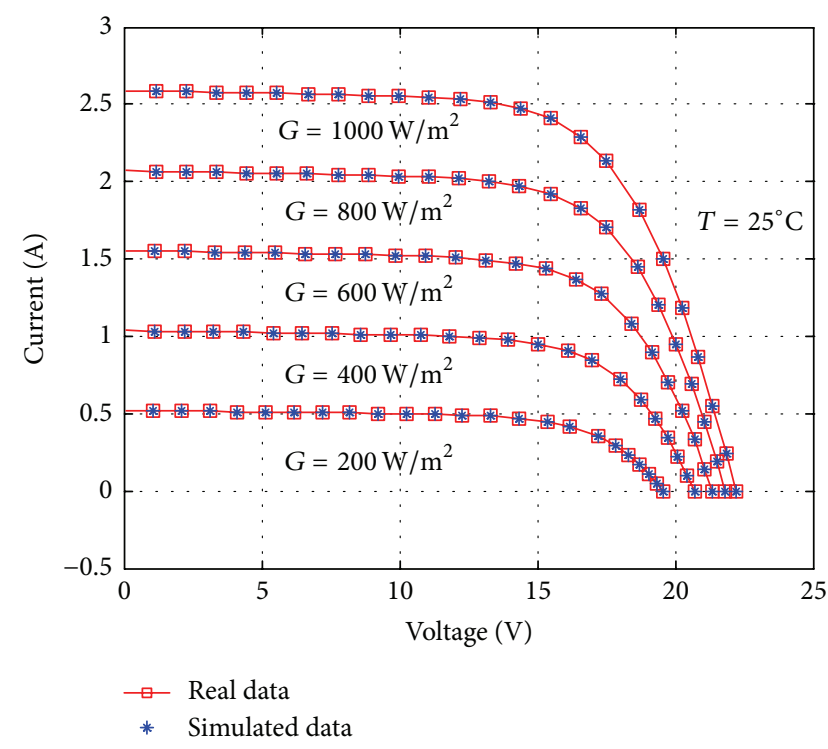

FIGURE 11: Comparison between real data and the simulated data by PCE for thin-film ST40 at different irradiances.

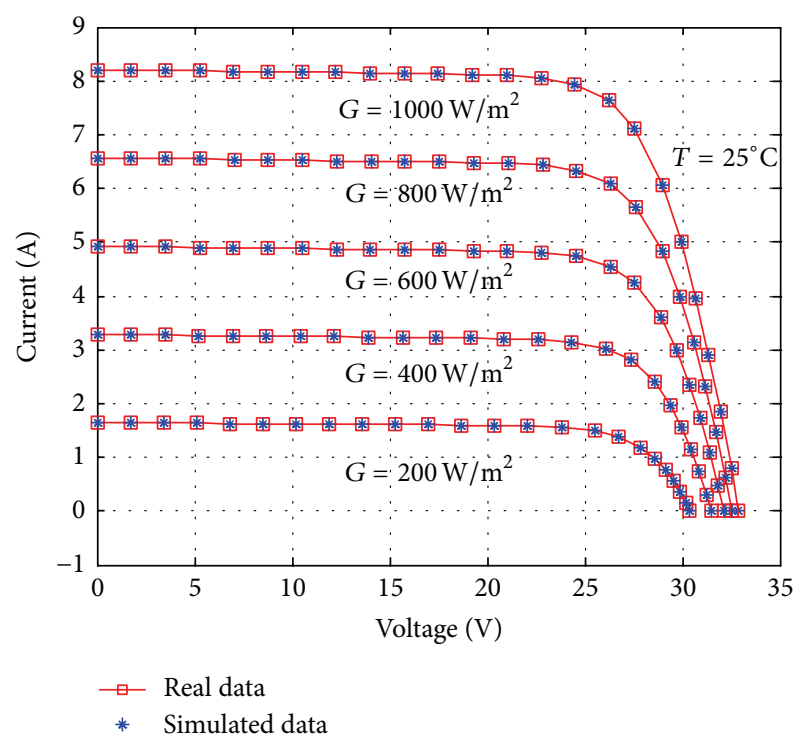

FIGURE 12: Comparison between real data and the simulated data by PCE for multicrystalline KC200GT at different irradiances.

the early stage PCE has a faster convergence speed compared with $R_{\mathrm{cr}}$-IJADE. In addition, PCE is tested using three different sources of data at different irradiance levels with constant temperature and at three different temperatures, with constant irradiance. From the experiment results, RMSE for various weather conditions reached the value of $10^{-5}$. Therefore, the parameter extraction performance of the proposed PCE technique is verified with good accuracy.

Therefore, the proposed PCE technique provides a good balance between exploration and exploitation. The new technique provides another optional method to extract parameters of solar cell models. In the future research, by mathematical analysis using a dynamic system, such as 
TABLE 13: The extracted parameters for monocrystalline SM55 PV module by PCE at different temperatures and values of irradiance of $1000 \mathrm{~W} / \mathrm{m}^{2}$.

\begin{tabular}{lccc}
\hline Parameters & \multicolumn{3}{c}{ Temperature } \\
& $\left(25^{\circ} \mathrm{C}\right)$ & $\left(40^{\circ} \mathrm{C}\right)$ & $\left(60^{\circ} \mathrm{C}\right)$ \\
\hline Monocrystalline SM55 & & & \\
$I_{\mathrm{ph}}(\mathrm{A})$ & 3.455710 & 3.490212 & 3.536219 \\
$I_{\text {sd }}(\mu \mathrm{A})$ & 0.00288916 & 0.02139623 & 0.23703510 \\
$R_{s}(\Omega)$ & 0.495620 & 0.495663 & 0.495628 \\
$R_{\text {sh }}(\Omega)$ & 299.9873 & 299.8248 & 299.8124 \\
$n$ & 1.123473 & 1.123268 & 1.123354 \\
RMSE & $4.82 E-05$ & $6.51 E-05$ & $4.73 E-05$ \\
\hline
\end{tabular}

TABLE 14: The extracted parameters for thin-film ST40 PV module by PCE at different temperatures and values of irradiance of $1000 \mathrm{~W} / \mathrm{m}^{2}$.

\begin{tabular}{lcccc}
\hline \multirow{2}{*}{ Parameters } & \multicolumn{5}{c}{ Temperature } \\
& $\left(25^{\circ} \mathrm{C}\right)$ & $\left(40^{\circ} \mathrm{C}\right)$ & $\left(55^{\circ} \mathrm{C}\right)$ & $\left(70^{\circ} \mathrm{C}\right)$ \\
\hline Thin-film ST40 & & & & \\
$I_{\mathrm{ph}}(\mathrm{A})$ & 2.596880 & 2.614915 & 2.632917 & 2.650986 \\
$I_{\text {sd }}(\mu \mathrm{A})$ & 2.789916 & 11.564938 & 42.589211 & 140.543289 \\
$R_{s}(\Omega)$ & 0.798689 & 0.799007 & 0.799080 & 0.799203 \\
$R_{\text {sh }}(\Omega)$ & 300.3258 & 299.6235 & 299.3048 & 297.4168 \\
$n$ & 1.499926 & 1.499275 & 1.499222 & 1.498911 \\
RMSE & $3.88 E-05$ & $3.40 E-05$ & $3.79 E-05$ & $8.17 E-05$ \\
\hline
\end{tabular}

TABLE 15: The extracted parameters for multicrystalline KC200GT PV module by PCE at different temperatures and values of irradiance of $1000 \mathrm{~W} / \mathrm{m}^{2}$.

\begin{tabular}{lccc}
\hline Parameters & \multicolumn{3}{c}{ Temperature } \\
& $\left(25^{\circ} \mathrm{C}\right)$ & $\left(50^{\circ} \mathrm{C}\right)$ & $\left(75^{\circ} \mathrm{C}\right)$ \\
\hline Multicrystalline KC200GT & & & \\
$I_{\text {ph }}(\mathrm{A})$ & 8.221126 & 8.301147 & 8.381114 \\
$I_{\text {sd }}(\mu \mathrm{A})$ & 0.00400505 & 0.107120 & 1.825925 \\
$R_{s}(\Omega)$ & 0.298004 & 0.298043 & 0.297969 \\
$R_{\text {sh }}(\Omega)$ & 220.0150 & 219.9471 & 220.0545 \\
$n$ & 1.423000 & 1.422846 & 1.423102 \\
RMSE & $5.58 E-05$ & $6.07 E-05$ & $2.99 E-05$ \\
\hline
\end{tabular}

the Markov chains, we are going to prove and explain the convergence of the proposed approach.

\section{Competing Interests}

The authors declare that they have no competing interests.

\section{Acknowledgments}

This research was supported by the National Natural Science Foundation of China (no. 61574038), the Science Foundation of Fujian Education Department (no. JK2014003 and no. JA14038), the Science Foundation of Fujian Science \& Technology Department (no. 2015H0021, nos. 2015J05124 and 2016H6012), and the Scientific Research Foundation for
TABLE 16: The extracted parameters for three different types of PV modules by PCE at different values of irradiance and temperatures of $25^{\circ} \mathrm{C}$.

\begin{tabular}{|c|c|c|c|}
\hline Parameters & $\begin{array}{c}\text { Monocrystalline } \\
\text { SM55 }\end{array}$ & $\begin{array}{l}\text { Thin-film } \\
\text { ST40 }\end{array}$ & $\begin{array}{c}\text { Multicrystalline } \\
\text { KC200GT }\end{array}$ \\
\hline \multicolumn{4}{|c|}{$G=1000 \mathrm{~W} / \mathrm{m}^{2}$} \\
\hline$I_{\mathrm{ph}}(\mathrm{A})$ & 3.455710 & 2.596880 & 8.221126 \\
\hline$I_{\text {sd }}(\mu \mathrm{A})$ & 0.00288916 & 2.789916 & 0.00400505 \\
\hline$R_{s}(\Omega)$ & 0.495620 & 0.798689 & 0.298004 \\
\hline$R_{\mathrm{sh}}(\Omega)$ & 299.9873 & 300.3258 & 220.0150 \\
\hline$n$ & 1.123473 & 1.499926 & 1.423000 \\
\hline RMSE & $4.82 E-05$ & $3.88 E-05$ & $5.58 E-05$ \\
\hline \multicolumn{4}{|c|}{$G=800 \mathrm{~W} / \mathrm{m}^{2}$} \\
\hline$I_{\mathrm{ph}}(\mathrm{A})$ & 2.764556 & 2.077492 & 6.576922 \\
\hline$I_{\text {sd }}(\mu \mathrm{A})$ & 0.00287531 & 2.776931 & 0.00399302 \\
\hline$R_{s}(\Omega)$ & 0.495699 & 0.799113 & 0.298074 \\
\hline$R_{\mathrm{sh}}(\Omega)$ & 307.4021 & 307.4427 & 225.5355 \\
\hline$n$ & 1.123210 & 1.499416 & 1.422798 \\
\hline RMSE & $2.32 E-05$ & $3.30 E-05$ & $5.03 E-05$ \\
\hline \multicolumn{4}{|c|}{$G=600 \mathrm{~W} / \mathrm{m}^{2}$} \\
\hline$I_{\mathrm{ph}}(\mathrm{A})$ & 2.073429 & 1.558121 & 4.932673 \\
\hline$I_{\mathrm{sd}}(\mu \mathrm{A})$ & 0.00287555 & 2.7814890 & 0.00400713 \\
\hline$R_{s}(\Omega)$ & 0.495743 & 0.798844 & 0.297980 \\
\hline$R_{\mathrm{sh}}(\Omega)$ & 329.4398 & 329.7704 & 242.4279 \\
\hline$n$ & 1.123214 & 1.499600 & 1.423032 \\
\hline RMSE & $3.07 E-05$ & $2.64 E-05$ & $2.94 E-05$ \\
\hline \multicolumn{4}{|c|}{$G=400 \mathrm{~W} / \mathrm{m}^{2}$} \\
\hline$I_{\mathrm{ph}}(\mathrm{A})$ & 1.382294 & 1.038787 & 3.288464 \\
\hline$I_{\mathrm{sd}}(\mu \mathrm{A})$ & 0.00287087 & 2.768818 & 0.00398943 \\
\hline$R_{s}(\Omega)$ & 0.495802 & 0.799834 & 0.298126 \\
\hline$R_{\mathrm{sh}}(\Omega)$ & 396.0824 & 395.9454 & 292.7323 \\
\hline$n$ & 1.123117 & 1.499072 & 1.422728 \\
\hline RMSE & $2.27 E-05$ & $3.08 E-05$ & $4.86 E-05$ \\
\hline \multicolumn{4}{|c|}{$G=200 \mathrm{~W} / \mathrm{m}^{2}$} \\
\hline$I_{\mathrm{ph}}(\mathrm{A})$ & 0.691135 & 0.519409 & 1.644224 \\
\hline$I_{s d}(\mu \mathrm{A})$ & 0.00289179 & 2.758105 & 0.00399516 \\
\hline$R_{s}(\Omega)$ & 0.495188 & 0.801411 & 0.298241 \\
\hline$R_{\mathrm{sh}}(\Omega)$ & 596.9193 & 595.3726 & 444.5314 \\
\hline$n$ & 1.123531 & 1.498570 & 1.422821 \\
\hline RMSE & $2.92 E-05$ & $3.18 E-05$ & $2.73 E-05$ \\
\hline
\end{tabular}

the Returned Overseas Chinese Scholars, State Education Ministry (no. LXKQ201504).

\section{References}

[1] X. Chen, K. Yu, W. Du, W. Zhao, and G. Liu, "Parameters identification of solar cell models using generalized oppositional teaching learning based optimization," Energy, vol. 99, pp. 170$180,2016$.

[2] D. Oliva, E. Cuevas, and G. Pajares, "Parameter identification of solar cells using artificial bee colony optimization," Energy, vol. 72, pp. 93-102, 2014. 
[3] A. Askarzadeh and A. Rezazadeh, "Artificial bee swarm optimization algorithm for parameters identification of solar cell models," Applied Energy, vol. 102, pp. 943-949, 2013.

[4] W. Gong and Z. Cai, "Parameter extraction of solar cell models using repaired adaptive differential evolution," Solar Energy, vol. 94, pp. 209-220, 2013.

[5] K. Ishaque, Z. Salam, H. Taheri, and A. Shamsudin, "A critical evaluation of EA computational methods for photovoltaic cell parameter extraction based on two diode model," Solar Energy, vol. 85, no. 9, pp. 1768-1779, 2011.

[6] A. Askarzadeh and L. dos Santos Coelho, "Determination of photovoltaic modules parameters at different operating conditions using a novel bird mating optimizer approach," Energy Conversion and Management, vol. 89, pp. 608-614, 2015.

[7] W. Han, H.-H. Wang, and L. Chen, "Parameters identification for photovoltaic module based on an improved artificial fish swarm algorithm," Scientific World Journal, vol. 2014, Article ID 859239, 12 pages, 2014.

[8] T. Easwarakhanthan, J. Bottin, I. Bouhouch, and C. Boutrit, "Nonlinear minimization algorithm for determining the solar cell parameters with microcomputers," International Journal of Solar Energy, vol. 4, no. 1, pp. 1-12, 1986.

[9] A. Ortiz-Conde, F. J. G. Sánchez, and J. Muci, "New method to extract the model parameters of solar cells from the explicit analytic solutions of their illuminated I-V characteristics," Solar Energy Materials and Solar Cells, vol. 90, no. 3, pp. 352-361, 2006.

[10] Q. Niu, L. Zhang, and K. Li, "A biogeography-based optimization algorithm with mutation strategies for model parameter estimation of solar and fuel cells," Energy Conversion and Management, vol. 86, pp. 1173-1185, 2014.

[11] M. Ye, X. Wang, and Y. Xu, "Parameter extraction of solar cells using particle swarm optimization," Journal of Applied Physics, vol. 105, no. 9, Article ID 094502, 2009.

[12] H. Wei, J. Cong, X. Lingyun, and S. Deyun, "Extracting solar cell model parameters based on chaos particle swarm algorithm," in Proceedings of the International Conference on Electric Information and Control Engineering (ICEICE '11), pp. 398-402, IEEE, Wuhan, China, April 2011.

[13] J. A. Jervase, H. Bourdoucen, and A. Al-Lawati, "Solar cell parameter extraction using genetic algorithms," Measurement Science and Technology, vol. 12, no. 11, pp. 1922-1925, 2001.

[14] M. Zagrouba, A. Sellami, M. Bouaïcha, and M. Ksouri, "Identification of PV solar cells and modules parameters using the genetic algorithms: application to maximum power extraction," Solar Energy, vol. 84, no. 5, pp. 860-866, 2010.

[15] M. R. AlRashidi, M. F. AlHajri, K. M. El-Naggar, and A. K. AlOthman, "A new estimation approach for determining the IV characteristics of solar cells," Solar Energy, vol. 85, no. 7, pp. 1543-1550, 2011.

[16] K. Ishaque and Z. Salam, "An improved modeling method to determine the model parameters of photovoltaic (PV) modules using differential evolution (DE)," Solar Energy, vol. 85, no. 9, pp. 2349-2359, 2011.

[17] L. L. Jiang, D. L. Maskell, and J. C. Patra, "Parameter estimation of solar cells and modules using an improved adaptive differential evolution algorithm," Applied Energy, vol. 112, pp. 185-193, 2013.

[18] K. Ishaque, Z. Salam, S. Mekhilef, and A. Shamsudin, "Parameter extraction of solar photovoltaic modules using penaltybased differential evolution," Applied Energy, vol. 99, pp. 297308, 2012.
[19] M. F. AlHajri, K. M. El-Naggar, M. R. AlRashidi, and A. K. Al-Othman, "Optimal extraction of solar cell parameters using pattern search," Renewable Energy, vol. 44, pp. 238-245, 2012.

[20] K. M. El-Naggar, M. R. AlRashidi, M. F. AlHajri, and A. K. Al-Othman, "Simulated Annealing algorithm for photovoltaic parameters identification," Solar Energy, vol. 86, no. 1, pp. 266274, 2012.

[21] A. Askarzadeh and A. Rezazadeh, "Parameter identification for solar cell models using harmony search-based algorithms," Solar Energy, vol. 86, no. 11, pp. 3241-3249, 2012.

[22] A. Askarzadeh and A. Rezazadeh, "A new heuristic optimization algorithm for modeling of proton exchange membrane fuel cell: bird mating optimizer," International Journal of Energy Research, vol. 37, no. 10, pp. 1196-1204, 2013.

[23] K. Katayama and H. Narihisa, "On fundamental design of parthenogenetic algorithm for the binary quadratic programming problem," in Proceedings of the IEEE Congress on Evolutionary Computation, pp. 356-363, May 2001.

[24] M. Barukčić, S. Nikolovski, and F. Jovíc, "Hybrid evolutionaryheuristic algorithm for capacitor banks allocation," Journal of Electrical Engineering, vol. 61, no. 6, pp. 332-340, 2010.

[25] J. Wu and $\mathrm{H}$. Wang, "A parthenogenetic algorithm for the founder sequence reconstruction problem," Journal of Computers, vol. 8, no. 11, pp. 2934-2941, 2013.

[26] R. Storn and K. Price, "Differential evolution-a simple and efficient heuristic for global optimization over continuous spaces," Journal of Global Optimization, vol. 11, no. 4, pp. 341359, 1997.

[27] S. Das, A. Abraham, U. K. Chakraborty, and A. Konar, "Differential evolution using a neighborhood-based mutation operator," IEEE Transactions on Evolutionary Computation, vol. 13, no. 3, pp. 526-553, 2009.

[28] W.-C. Yeh, "Orthogonal simplified swarm optimization for the series-parallel redundancy allocation problem with a mix of components," Knowledge-Based Systems, vol. 64, pp. 1-12, 2014.

[29] J. Brest, S. Greiner, B. Bošković, M. Mernik, and V. Zumer, "Self-adapting control parameters in differential evolution: a comparative study on numerical benchmark problems," IEEE Transactions on Evolutionary Computation, vol. 10, no. 6, pp. 646-657, 2006.

[30] X.-S. Yang and S. Deb, "Cuckoo search via Lévy flights," in Proceedings of the World Congress on Nature \& Biologically Inspired Computing (NaBIC '09), pp. 210-214, Coimbatore, India, December 2009.

[31] D. Karaboga and B. Basturk, "A powerful and efficient algorithm for numerical function optimization: artificial bee colony (ABC) algorithm," Journal of Global Optimization, vol. 39, no. 3, pp. 459-471, 2007.

[32] P. N. Suganthan, N. Hansen, J. J. Liang et al., "Problem definitions and evaluation criteria for the CEC 2005 special session on real-parameter optimization," KanGAL Report 2005005, 2005.

[33] SHELL, Shell SM55 photovoltaic solar module, http://www .solarquest.com/microsolar/suppliers/siemens/sm55.pdf.

[34] Characteristics of a PV module PV module: shell solar, ST 40, http://ohodas.opx.pl/shell-st40-solar-panel.php.

[35] Kyocera, KC200GT, high efficiency multicrystal photovoltaic module, 2012, http://www.kyocerasolar.com/assets/001/5195 .pdf. 

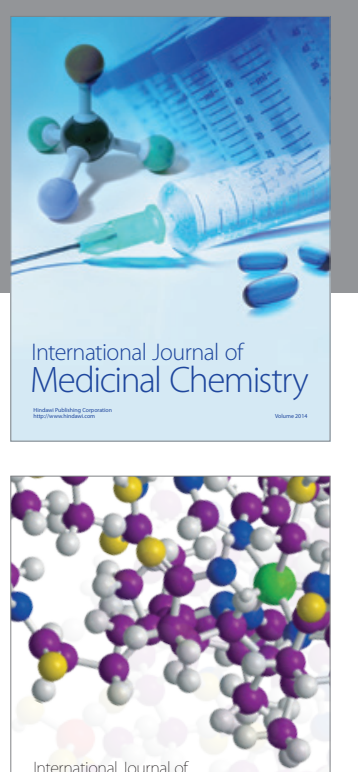

Carbohydrate Chemistry

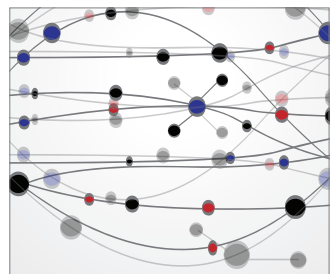

The Scientific World Journal
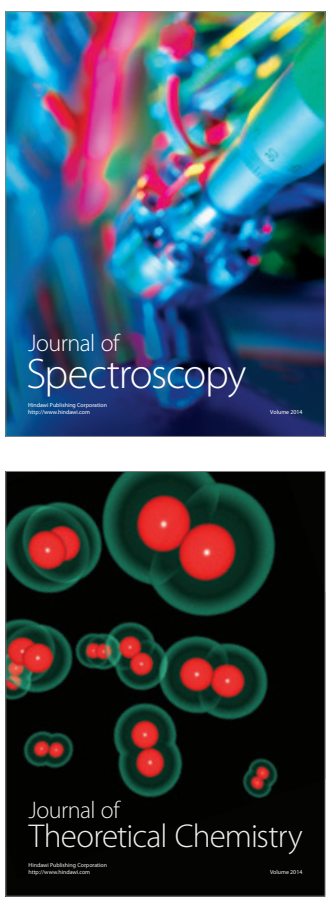
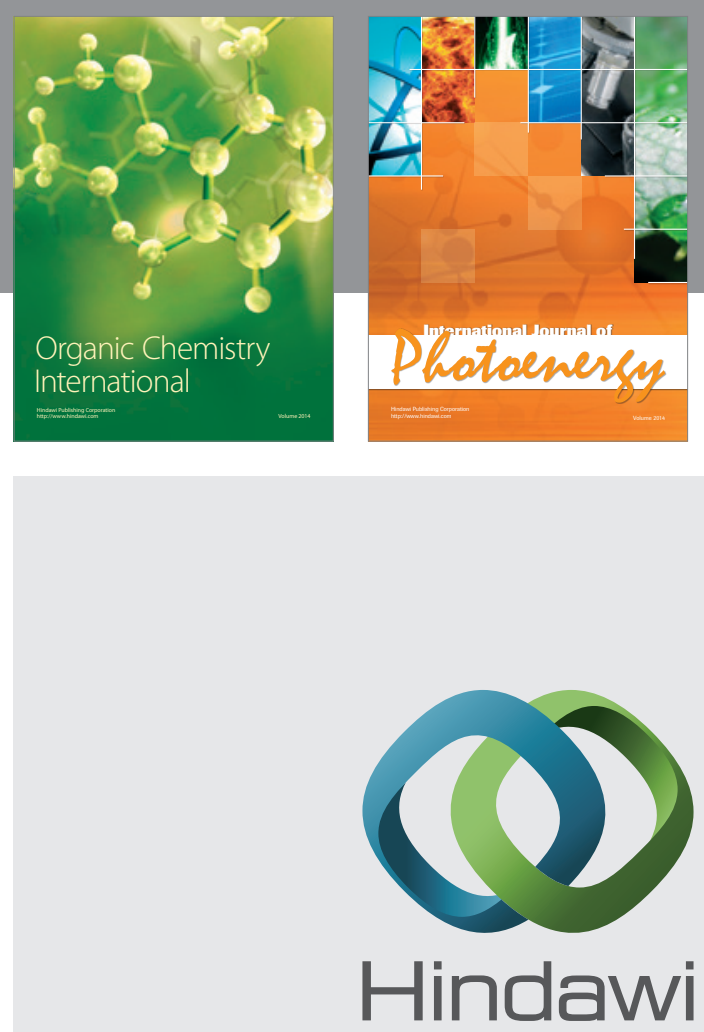

Submit your manuscripts at

http://www.hindawi.com

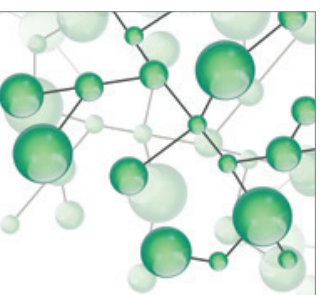

International Journal of

Inorganic Chemistry

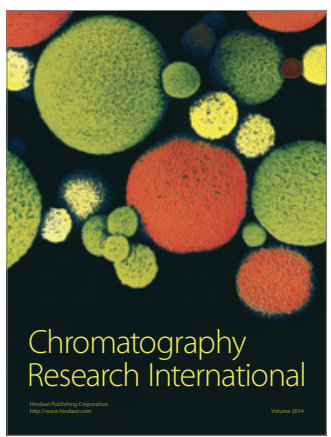

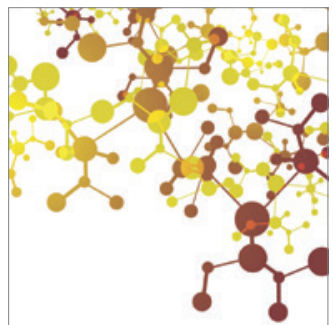

Applied Chemistry
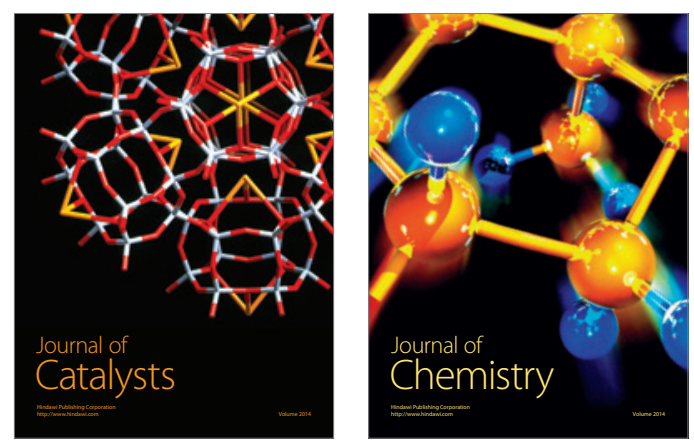
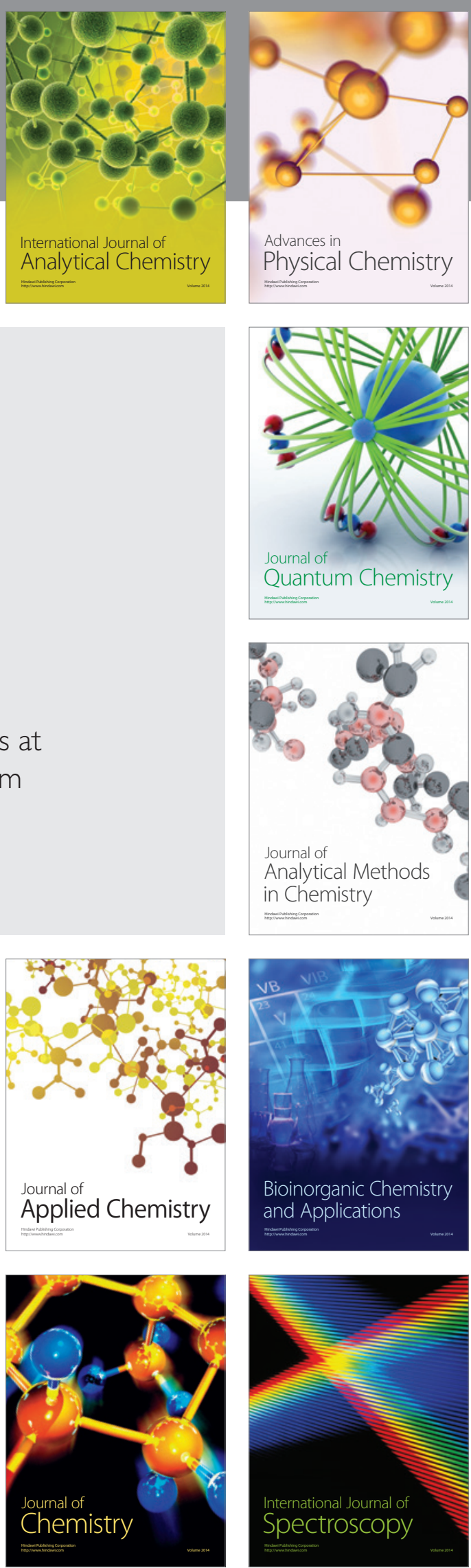